\title{
Interval-Valued Intuitionistic Fuzzy Derivative and Differential Operations
}

\author{
Hua Zhao \\ Institute of Sciences, PLA University of Science and Technology \\ Nanjing, Jiangsu 211101, China \\ Zeshui Xu* \\ Business School, Sichuan University, Chengdu, Sichuan 610064, China \\ Zeqing Yao \\ Institute of Sciences, PLA University of Science and Technology \\ Nanjing, Jiangsu 211101, China
}

Received 16 September 2015

Accepted 29 October 2015

\begin{abstract}
The interval-valued intuitionistic fuzzy set (IVIFS) generalizes Atanassov's intuitionistic fuzzy set (A-IFS) with the membership and non-membership degrees being intervals instead of real numbers, so it can contain more information. In this paper, we study the derivatives and differentials under interval-valued intuitionistic fuzzy environment. Firstly, we discuss the four change directions (the addition, subtraction, multiplication and division directions) of the interval- valued intuitionistic fuzzy values (IVIFVs); Secondly, we propose four kinds of limits (the addition, subtraction, multiplication and division limits) for different sequences of IVIFVs, and then we define the concepts of interval-valued intuitionistic fuzzy function (IVIFF) and study the continuities of IVIFFs; Thirdly, we develop two kinds of derivatives (the subtraction and division derivatives) of IVIFFs and give an equivalent condition for the existence of the derivative of an IVIFF. At last, we define the concepts of two kinds of differentials (the subtraction and division differentials) of IVIFFs and discuss the approximate computations of IVIFFs by the developed differentials.
\end{abstract}

Keywords: Interval-valued intuitionistic fuzzy set (IVIFS); Interval-valued intuitionistic fuzzy function (IVIFF); Limit; Continuity; Derivative; Differential.

\section{Introduction}

As an important generalization of fuzzy set ${ }^{1}$, Atanassov's intuitionistic fuzzy set (A-IFS) ${ }^{2}$ has attracted lots of attention. It is added a degree of hesitance compared to the classic fuzzy sets for characterizing the uncertainty in humans' consciousness. Up to now, it has been applied in many fields, such as decision making ${ }^{3-5}$, clustering ${ }^{6-7}$, medical diagnosis ${ }^{8}$, image fusion ${ }^{9}$, and so on. A large number of research results under intuitionistic fuzzy environment have been derived from various directions, such as intuitionistic fuzzy probability ${ }^{10}$, intuitionistic fuzzy approximate reasoning ${ }^{11}$ and intuitionistic fuzzy algebra ${ }^{12-13}$ etc. Recently, Lei \& $\mathrm{Xu}^{14}$ discussed the generalizations of derivative and differential under intuitionistic fuzzy environment, obtained some useful results and pointed

\footnotetext{
* Corresponding author. Tel.: +8625 84483382. E-mails: xuzeshui@263.net; zhaohua_pla@163.com
} 
out a new direction for the study of infinitesimal calculus. Lei \& $\mathrm{Xu}{ }^{15}$ further studied the definite integral of intuitionistic fuzzy functions (IFFs), and gave the Newton-Leibniz formula under intuitionistic fuzzy environment, and then discussed the basic properties of intuitionistic fuzzy calculus. Lei et.al ${ }^{16}$ proposed a series of general integrals to aggregate continuous intuitionistic fuzzy information based on Archimedean t-conorm and t-norm. Apart from the above researches, $\mathrm{Xu}$ and Yager ${ }^{17}$ first proposed the concept of intuitionistic fuzzy value (IFV) and gave the operations of IFVs. Some authors paid attention to the methods of ranking IFVs in Refs. ${ }^{18-20}$. Moreover, Atanassov presented the interval-valued intuitionistic fuzzy set (IVIFS) ${ }^{21}$ whose membership degree and nonmembership degree are all intervals instead of two real numbers aiming at the case where the membership degree and the non- membership degree cannot be given conveniently by crisp numbers.

By using the theory of IVIFSs, many scholars have put forward an amount of methods dealing with interval-valued intuitionistic fuzzy information in various fields, including decision making 22-23, information fusion ${ }^{24-25}$, linear programming ${ }^{26}$, AHP ${ }^{27}$, and so on. Furthermore, the rankings of interval-valued intuitionistic fuzzy values (IVIFVs) have also attracted attention in Refs. 28-29. By introducing parameters, Zhang et al. ${ }^{30}$ generalized the IVIFS into a new one which was proven to be a closed algebraic system as the IFS and the IVIFS. However, no one has so far attempted to study the derivative and differential of IVIFSs, which are very necessary for further developing the theory of IVIFSs. In this paper, we shall focus on investigating this issue. To do that, we give some preparations for the whole work in Section 2, and define the concepts of the change values of IVIFVs in Section 3. Then, we define the concept of convergence of sequences of IVIFVs and give a necessary and sufficient condition for the convergences of sequences of IVIFVs in Section 4. Moreover, we discuss the continuity and differentiability of interval-valued intuitionistic fuzzy functions (IVIFFs) in Section 5, and explore the differentials of IVIFFs in Section 6. Finally, we conclude the paper in Section 7.

\section{Preliminaries}

As a preparation for further discussions, we first review the related concepts and operations about IVIFSs and IVIFVs.

Atanassov and Gargov ${ }^{21}$ defined the concept of interval-valued intuitionistic fuzzy set (IVIFS) as follows:

Definition $1^{21}$. An IVIFS $\tilde{A}$ over $X$ is an object having the form:

$$
\widetilde{A}=\left\{<x, \widetilde{\mu}_{\widetilde{A}}(x), \widetilde{v}_{\widetilde{A}}(x)>\mid x \in X\right\}
$$

where $\tilde{\mu}_{\widetilde{A}}(x) \subset[0,1]$ and $\widetilde{v}_{\widetilde{A}}(x) \subset[0,1]$ are intervals, and for each $x \in X$ :

$$
\sup \tilde{\mu}_{\widetilde{A}}(x)+\sup {\widetilde{v_{\widetilde{A}}}}_{\widetilde{A}}(x) \leq 1
$$

Especially, if each of the intervals $\tilde{\mu}_{\widetilde{A}}(x)$ and $\widetilde{v}_{\widetilde{A}}(x)$ contains only one number, i.e., if for any $x \in X$ :

$$
\begin{aligned}
\mu_{\widetilde{A}}(x) & =\inf \widetilde{\mu}_{\widetilde{A}}(x)=\sup \widetilde{\mu}_{\widetilde{A}}(x) \\
v_{\widetilde{A}}(x) & =\inf \widetilde{v}_{\widetilde{A}}(x)=\sup \widetilde{v}_{\widetilde{A}}(x)
\end{aligned}
$$

Then the given IVIFS $\tilde{A}$ is reduced to an ordinary intuitionistic fuzzy set (IFS) ${ }^{2}$.

On the basis of IVIFS, $\mathrm{Xu}{ }^{28}$ introduced the notion of interval-valued intuitionistic fuzzy value (IVIFV):

Definition $2{ }^{28}$. Suppose that $\tilde{A}=\left\{\left\langle x, \widetilde{\mu}_{\tilde{A}}(x), \widetilde{v}_{\widetilde{A}}(x)\right\rangle \mid x \in X\right\}$ is an IVIFS, then the pair $\left(\widetilde{\mu}_{\widetilde{A}}(x), \widetilde{v}_{\widetilde{A}}(x)\right)$ is called an IVIFV.

$$
\begin{gathered}
\mathrm{Xu}^{28} \text { expressed an IVIFV as }([a, b],[c, d]) \text {, where } \\
{[a, b] \subset[0,1],[c, d] \subset[0,1], b+d \leq 1}
\end{gathered}
$$

and let $\Theta$ be the set of all IVIFVs. Then $\mathrm{Xu}^{28}$ gave some operations of IVIFVs:

Definition $3{ }^{28}$. Let $\tilde{\alpha}_{1}=\left(\left[a_{1}, b_{1}\right],\left[c_{1}, d_{1}\right]\right)$ and $\tilde{\alpha}_{2}=\left(\left[a_{2}, b_{2}\right],\left[c_{2}, d_{2}\right]\right)$ be any two IVIFVs, then

(1) $\tilde{\alpha}_{1} \oplus \tilde{\alpha}_{2}=\left(\left[a_{1}+a_{2}-a_{1} a_{2}, b_{1}+b_{2}-b_{1} b_{2}\right],\left[c_{1} c_{2}, d_{1} d_{2}\right]\right)$;

(2) $\tilde{\alpha}_{1} \otimes \tilde{\alpha}_{2}=\left(\left[a_{1} a_{2}, b_{1} b_{2}\right],\left[c_{1}+c_{2}-c_{1} c_{2}, d_{1}+d_{2}-d_{1} d_{2}\right]\right)$;

(3) $\lambda \tilde{\alpha}_{1}=\left(\left[1-\left(1-a_{1}\right)^{\lambda}, 1-\left(1-b_{1}\right)^{\lambda}\right],\left[c_{1}^{\lambda}, d_{1}^{\lambda}\right]\right), \lambda>0$;

(4) $\tilde{\alpha}_{1}^{\lambda}=\left(\left[a_{1}^{\lambda}, b_{1}^{\lambda}\right],\left[1-\left(1-c_{1}\right)^{\lambda}, 1-\left(1-d_{1}\right)^{\lambda}\right]\right), \lambda>0$.

All the above computing results are also IVIFVs ${ }^{28}$. Based on Definition 3, Xu ${ }^{28}$ further verified the operation laws as follows:

Proposition $1{ }^{28}$. Let $\tilde{\alpha}_{1}=\left(\left[a_{1}, b_{1}\right],\left[c_{1}, d_{1}\right]\right)$ and $\tilde{\alpha}_{2}=\left(\left[a_{2}, b_{2}\right],\left[c_{2}, d_{2}\right]\right)$ be arbitrary two IVIFVs, then 
(1) $\tilde{\alpha}_{1} \oplus \tilde{\alpha}_{2}=\tilde{\alpha}_{2} \oplus \tilde{\alpha}_{1}$

(2) $\tilde{\alpha}_{1} \otimes \tilde{\alpha}_{2}=\tilde{\alpha}_{2} \otimes \tilde{\alpha}_{1}$;

(3) $\lambda\left(\tilde{\alpha}_{1} \oplus \tilde{\alpha}_{2}\right)=\lambda \tilde{\alpha}_{1} \oplus \lambda \tilde{\alpha}_{2}, \lambda \geq 0$;

(4) $\left(\tilde{\alpha}_{1} \otimes \tilde{\alpha}_{2}\right)^{\lambda}=\tilde{\alpha}_{1}^{\lambda} \otimes \tilde{\alpha}_{2}^{\lambda}, \lambda \geq 0$;

(5) $\lambda_{1} \tilde{\alpha}_{1} \oplus \lambda_{2} \tilde{\alpha}_{1}=\left(\lambda_{1}+\lambda_{2}\right) \tilde{\alpha}_{1}, \lambda_{1}, \lambda_{2} \geq 0$;

(6) $\tilde{\alpha}_{1}^{\lambda_{1}} \otimes \tilde{\alpha}_{1}^{\lambda_{2}}=\left(\tilde{\alpha}_{1}\right)^{\lambda_{1}+\lambda_{2}}, \lambda_{1}, \lambda_{2} \geq 0$.

In order to investigate the derivative and differential operations of IVIFVs, we should first define the subtraction and division operations of IVIFVs. Motivated by the subtraction and division operations of IFVs ${ }^{14}$, below we define these two basic operations of IVIFVs:

Definition 4. Let $\tilde{\alpha}_{1}=\left(\left[a_{1}, b_{1}\right],\left[c_{1}, d_{1}\right]\right)$ and $\tilde{\alpha}_{2}=\left(\left[a_{2}, b_{2}\right],\left[c_{2}, d_{2}\right]\right)$ be two given IVIFVs, then

(1) The subtraction operation of IVIFVs is defined as follows:

$$
\tilde{\alpha}_{1} \ominus \tilde{\alpha}_{2}=\left\{\begin{array}{cc}
\left(\left[\frac{a_{1}-a_{2}}{1-a_{2}}, \frac{b_{1}-b_{2}}{1-b_{2}}\right],\left[\frac{c_{1}}{c_{2}}, \frac{d_{1}}{d_{2}}\right]\right), & \begin{array}{r}
\text { if } a_{1} \geq a_{2}, b_{1} \geq b_{2}, c_{1} \leq c_{2}, d_{1} \leq d_{2} \\
\text { and } c_{2}>0, d_{2}>0 \\
\text { and } c_{1}\left(1-a_{2}\right) \leq c_{2}\left(1-a_{1}\right), \\
d_{1}\left(1-b_{2}\right) \leq d_{2}\left(1-b_{1}\right)
\end{array} \\
([0,0],[1,1]), & \text { otherwise }
\end{array}\right.
$$

(2) The division operation of IVIFVs has the following forms:

$$
\tilde{\alpha}_{1} \oslash \tilde{\alpha}_{2}=\left\{\begin{array}{cc}
\left(\left[\frac{a_{1}}{a_{2}}, \frac{b_{1}}{b_{2}}\right],\left[\frac{c_{1}-c_{2}}{1-c_{2}}, \frac{d_{1}-d_{2}}{1-d_{2}}\right]\right), & \begin{array}{c}
\text { if } a_{1} \leq a_{2}, b_{1} \leq b_{2}, c_{1} \geq c_{2}, d_{1} \geq d_{2} \\
\text { and } a_{2}>0, b_{2}>0 \\
\text { and } a_{1}\left(1-c_{2}\right) \leq a_{2}\left(1-c_{1}\right), \\
b_{1}\left(1-d_{2}\right) \leq b_{2}\left(1-d_{1}\right)
\end{array} \\
([0,0],[1,1]), & \text { otherwise }
\end{array}\right.
$$

By Definitions 3 and 4, we can easily verify that the inverse operation of " $\oplus$ " is the operation " $\ominus$ ", that is to say, if $\tilde{\alpha}$ and $\tilde{\beta}$ are IVIFVs, then $\tilde{\alpha} \oplus \tilde{\beta} \ominus \tilde{\beta}=\tilde{\alpha}$. Similarly the division operation " $\oslash$ " is the inverse of the multiplication operation " $\otimes$ ".

Enlightened by the partially ordered set $\left(L, \leq_{L}\right)$ put forward by Deschrijver and Kerre ${ }^{31}$, we develop the following simple method for comparing any two IVIFVs:

Definition 5. Let $\tilde{\alpha}_{1}=\left(\left[a_{1}, b_{1}\right],\left[c_{1}, d_{1}\right]\right) \quad$ and $\tilde{\alpha}_{2}=\left(\left[a_{2}, b_{2}\right],\left[c_{2}, d_{2}\right]\right)$ be two IVIFVs, then
(1) If $a_{1} \geq a_{2}, b_{1} \geq b_{2}$ and $c_{1} \leq c_{2}, d_{1} \leq d_{2}$, then $\tilde{\alpha}_{1} \geq_{L} \tilde{\alpha}_{2}$

(2) If $a_{1} \leq a_{2}, b_{1} \leq b_{2}$ and $c_{1} \geq c_{2}, d_{1} \geq d_{2}$, then $\tilde{\alpha}_{1} \leq_{L} \tilde{\alpha}_{2}$

(3) If $a_{1}=a_{2}, b_{1}=b_{2}$ and $c_{1}=c_{2}, d_{1}=d_{2}$, then $\tilde{\alpha}_{1}=\tilde{\alpha}_{2}$.

Additionally, we introduce two common aggregation techniques for IVIFVs ${ }^{28}$ :

Definition $6{ }^{28}$. Assume that $\tilde{\alpha}_{i}=\left(\left[a_{i}, b_{i}\right],\left[c_{i}, d_{i}\right]\right)$ $(j=1,2, \ldots, n)$ are a collection of IVIFVs, and let IIFWA: $\Theta^{n} \rightarrow \Theta$, then the function:

$$
\operatorname{IIFWA}_{\omega}\left(\tilde{\alpha}_{1}, \tilde{\alpha}_{2}, \ldots, \tilde{\alpha}_{n}\right)=\bigoplus_{j=1}^{n}\left(\omega_{j} \tilde{\alpha}_{j}\right)
$$

is called an IIFWA operator, where $\omega=\left(\omega_{1}, \omega_{2}, \ldots, \omega_{n}\right)^{T}$ is the weight vector of $\tilde{\alpha}_{j}(j=1,2, \ldots, n)$, with $\omega_{j} \geq 0$, $(j=1,2, \ldots, n), \sum_{j=1}^{n} \omega_{j}=1$, and the aggregated result by the IIFWA operator is an IVIFV:

$$
\begin{aligned}
& \operatorname{IIFWA}_{\omega}\left(\tilde{\alpha}_{1}, \tilde{\alpha}_{2}, \ldots, \tilde{\alpha}_{n}\right)= \\
& \qquad\left(\left[1-\prod_{j=1}^{n}\left(1-a_{j}\right)^{\omega_{j}}, 1-\prod_{j=1}^{n}\left(1-b_{j}\right)^{\omega_{j}}\right],\left[\prod_{j=1}^{n} c_{j}^{\omega_{j}}, \prod_{j=1}^{n} d_{j}^{\omega_{j}}\right]\right)
\end{aligned}
$$

Definition $7{ }^{28}$. Suppose that $\tilde{\alpha}_{i}=\left(\left[a_{i}, b_{i}\right],\left[c_{i}, d_{i}\right]\right)$ $(j=1,2, \ldots, n)$ are a set of IVIFVs, let IIFWG: $\Theta^{n} \rightarrow \Theta$, then the function:

$$
\operatorname{IIFWG}_{\omega}\left(\tilde{\alpha}_{1}, \tilde{\alpha}_{2}, \ldots, \tilde{\alpha}_{n}\right)=\bigotimes_{j=1}^{n} \tilde{\alpha}_{j}^{\omega_{j}}
$$

is called an IIFWG operator, where $\omega=\left(\omega_{1}, \omega_{2}, \ldots, \omega_{n}\right)^{T}$ is the weight vector of $\tilde{\alpha}_{j}(j=1,2, \ldots, n)$, with $\omega_{j} \geq 0,(j=1,2, \ldots, n)$, $\sum_{j=1}^{n} \omega_{j}=1$, and the integrated value by the IIFWG operator is also an IVIFV:

$$
\begin{aligned}
& \operatorname{IIFWG}_{\omega}\left(\tilde{\alpha}_{1}, \tilde{\alpha}_{2}, \ldots, \tilde{\alpha}_{n}\right)= \\
& \quad\left(\left[\prod_{j=1}^{n} a_{j}^{\omega_{j}}, \prod_{j=1}^{n} b_{j}^{\omega_{j}}\right],\left[1-\prod_{j=1}^{n}\left(1-c_{j}\right)^{\omega_{j}}, 1-\prod_{j=1}^{n}\left(1-d_{j}\right)^{\omega_{j}}\right]\right)
\end{aligned}
$$

\section{The change values of IVIFVs}

We all know that any two real numbers can be expressed for each other almost unconditionally by their 
basic operations: addition, subtraction, multiplication and division in real number field. While in intuitionistic fuzzy number field, any two IFVs can only be expressed for each other by their four basic operations under certain conditions ${ }^{14}$. After introducing the four basic operations of IVIFVs in Section 2, we naturally want to know what will happen in the field of IVIFVs. In the following, we will investigate this issue. We first give the notation of change values of IVIFVs:

Definition 8. Let $\tilde{\alpha}, \tilde{\alpha}_{0}$ and $\tilde{\beta}$ be three IVIFVs, if $\tilde{\alpha}=\tilde{\alpha}_{0} \diamond \tilde{\beta}$, and $\diamond \in\{\oplus, \otimes, \ominus, \oslash\}$, then we call $\tilde{\alpha}$ the change value of $\tilde{\alpha}_{0}$.

Below, we shall set about finding out in what conditions two IVIFVs can be expressed for each other by the addition, subtraction, multiplication and division operations. Now we consider the addition operation:

Suppose that $\tilde{\alpha}_{0}$ is a given IVIFV and $\tilde{\beta}$ is an arbitrary IVIFV, we are concerned about the value of $\tilde{\alpha}_{0} \oplus \tilde{\beta}$, and discuss it from the following two aspects:

(1) If $\tilde{\beta}=([0,0],[1,1])$, then it is clear that $\tilde{\alpha}_{0} \oplus \tilde{\beta}=\tilde{\alpha}_{0}$.

(2) If $\tilde{\beta} \neq([0,0],[1,1])$, then we let $\tilde{\alpha}_{0} \oplus \tilde{\beta}=\tilde{\alpha}$, where $\tilde{\alpha}_{0}=\left(\left[a_{0}, b_{0}\right],\left[c_{0}, d_{0}\right]\right)$ and $\tilde{\alpha}=([a, b],[c, d])$.

Considering that $\oplus$ is the inverse operation of the operation $\Theta$, we get $\tilde{\beta}=\tilde{\alpha} \ominus \tilde{\alpha}_{0}$. Because $\tilde{\beta} \neq([0,0],[1,1])$, then by the operation $\ominus$ of IVIFVs, there're some constraints between $\tilde{\alpha}_{0}$ and $\tilde{\alpha}$ :

$$
a \geq a_{0}, c \leq c_{0}, c_{0}>0, c\left(1-a_{0}\right) \leq c_{0}(1-a)
$$

and

$$
b \geq b_{0}, d \leq d_{0}, d_{0}>0, d\left(1-b_{0}\right) \leq d_{0}(1-b)
$$

For a given IVIFV $\tilde{\alpha}_{0}$, we collect all the IVIFVs $\tilde{\alpha}$ satisfying the above constraints into a set and denote it by $\tilde{A}_{\tilde{\alpha}_{0}}^{\oplus}$ :

$\tilde{f}_{\tilde{d}_{0}}=\left\{\tilde{\alpha} a \geq \geq a_{0}, b \geq b_{0}, c \leq c_{0}, d \leq d_{0}, c_{0}>0, d_{0}>0, c\left(1-a_{0}\right) \leq c_{0}(1-a), d\left(1-b_{0}\right) \leq d_{0}(1-b)\right\}$

In fact, $\tilde{A}_{\tilde{\alpha}_{0}}^{\oplus}$ can also be expressed by

$$
\tilde{A}_{\tilde{\alpha}_{0}}^{\oplus}=\left\{\tilde{\alpha} \mid \tilde{\alpha}=\tilde{\alpha}_{0} \oplus \tilde{\beta}, \forall \tilde{\beta} \in \Theta\right\}
$$

where $\forall$ means "for arbitrary". In other words, in the set $\tilde{A}_{\tilde{\alpha}_{0}}^{\oplus}$, the IVIFVs $\tilde{\alpha}_{0}$ and $\tilde{\alpha}$ can be expressed for each other by the addition operation of IVIFVs.

Similarly, we can get
(1) $\tilde{A}_{\tilde{\alpha}_{0}}^{\ominus}=\left\{\tilde{\alpha} \mid \tilde{\alpha}=\tilde{\alpha}_{0} \ominus \tilde{\beta}, \forall \tilde{\beta} \in \Theta\right\}$

and

$\tilde{A}_{\tilde{c}_{0}}=\left\{\tilde{\alpha} \mid a \leq a_{0}, b \leq b_{0}, c \geq c_{0}, d \geq d_{0}, c>0, d>0, c_{0}(1-a) \leq c\left(1-a_{0}\right), d_{0}(1-b) \leq d\left(1-b_{0}\right)\right\}$

(2) $\tilde{A}_{\tilde{\alpha}_{0}}^{\otimes}=\left\{\tilde{\alpha} \mid \tilde{\alpha}=\tilde{\alpha}_{0} \otimes \tilde{\beta}, \forall \tilde{\beta} \in \Theta\right\}$

and

$\tilde{f}_{\tilde{c}_{0}}^{\mathscr{Q}}=\left\{\tilde{\alpha} a \leq c_{0}, b \leq b_{0}, c \geq c_{0}, d \geq d_{0}, q_{0}>0, b_{0}>0, a\left(1-c_{0}\right) \leq a_{0}(1-c), b\left(1-d_{0}\right) \leq b_{0}(1-d)\right\}$

(3) $\tilde{A}_{\tilde{\alpha}_{0}}^{\ominus}=\left\{\tilde{\alpha} \mid \tilde{\alpha}=\tilde{\alpha}_{0} \oslash \tilde{\beta}, \forall \tilde{\beta} \in \Theta\right\}$

and

$\tilde{A}_{\alpha_{0}}=\left\{\tilde{\alpha} a_{0} \leq a, b_{0} \leq b, c_{0} \geq c, d_{0} \geq d, a>0, b>0, a_{0}(1-c) \leq a\left(1-c_{0}\right), b_{0}(1-d) \leq b\left(1-d_{0}\right)\right\}$

Definition 9. We call $\tilde{A}_{\tilde{\alpha}_{0}}^{\oplus}$ the addition region of $\tilde{\alpha}_{0}$, $\tilde{A}_{\tilde{\alpha}_{0}}^{\ominus}$ the subtraction region of $\tilde{\alpha}_{0}, \tilde{A}_{\tilde{\alpha}_{0}}^{\otimes}$ the multiplication region of $\tilde{\alpha}_{0}$, and $\tilde{A}_{\tilde{\alpha}_{0}}^{\ominus}$ the division region of $\tilde{\alpha}_{0}$, respectively.

The relations of these four sets $\tilde{A}_{\tilde{\alpha}_{0}}^{\oplus}, \tilde{A}_{\tilde{\alpha}_{0}}^{\ominus}, \tilde{A}_{\tilde{\alpha}_{0}}^{\otimes}$, and $\tilde{A}_{\tilde{\alpha}_{0}}^{\ominus}$ derived from $\tilde{\alpha}_{0}$ and the set of all the IVIFVs $\Theta$ can be shown by the following figure:

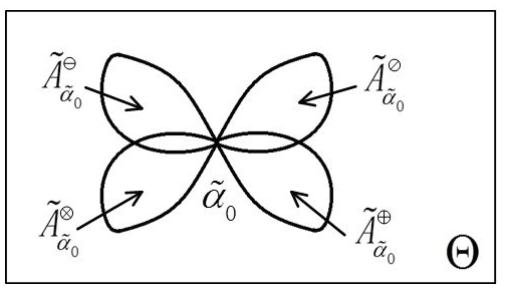

Firgue 1. The relations of the addition, subtraction, multiplication and division regions

Based on the above results, we give the following definition:

Definition 10. Assume that $\tilde{\alpha}_{1}, \tilde{\alpha}_{2}, \tilde{\alpha}_{3}$ and $\tilde{\alpha}_{4}$ are four IVIFVs. If $\tilde{\alpha}_{1} \in \tilde{A}_{\tilde{\alpha}_{0}}^{\oplus}, \tilde{\alpha}_{2} \in \tilde{A}_{\tilde{\alpha}_{0}}^{\ominus}, \tilde{\alpha}_{3} \in \tilde{A}_{\tilde{\alpha}_{0}}^{\otimes}$, and $\tilde{\alpha}_{4} \in \tilde{A}_{\tilde{\alpha}_{0}}^{\ominus}$, then $\tilde{\alpha}_{1}$ is called an addition change value for $\tilde{\alpha}_{0}, \tilde{\alpha}_{2}$ a subtraction change value for $\tilde{\alpha}_{0}, \tilde{\alpha}_{3}$ a multiplication change value for $\tilde{\alpha}_{0}$, and $\tilde{\alpha}_{4}$ a division change value for $\tilde{\alpha}_{0}$, respectively.

If $\tilde{\alpha}_{1} \in \tilde{A}_{\tilde{\alpha}_{0}}^{\oplus}, \quad \tilde{\alpha}_{2} \in \tilde{A}_{\tilde{\alpha}_{0}}^{\ominus}, \quad \tilde{\alpha}_{3} \in \tilde{A}_{\tilde{\alpha}_{0}}^{\otimes}$ and $\tilde{\alpha}_{4} \in \tilde{A}_{\tilde{\alpha}_{0}}^{\ominus}$, then there must exist the IVIFVs $\tilde{\beta}_{1}, \tilde{\beta}_{2}, \tilde{\beta}_{3}$ and $\tilde{\beta}_{4}$, such that $\tilde{\alpha}_{1}=\tilde{\alpha}_{0} \oplus \tilde{\beta}_{1}, \tilde{\alpha}_{2}=\tilde{\alpha}_{0} \ominus \tilde{\beta}_{2}, \tilde{\alpha}_{3}=\tilde{\alpha}_{0} \otimes \tilde{\beta}_{3}$, 
and $\tilde{\alpha}_{4}=\tilde{\alpha}_{0} \oslash \tilde{\beta}_{4}$. Therefore, an IVIFV has four change directions which can be shown visually by Figure 2 . This is quite different from a real number which has only two change directions.

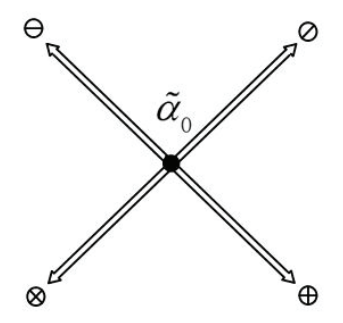

Figure 2. The four different change directions

\section{The sequences of IVIFVs}

\subsection{Various sequences of IVIFVs}

Definition 11. Suppose that $\left\{\tilde{\alpha}_{n}\right\} \quad(n=1,2, \cdots)$ are a sequence of IVIFVs, that is to say, every $\tilde{\alpha}_{n}$ is an IVIFV in the sequence. If $\exists N \in N^{+}$, and $\forall n>N$, $\tilde{\alpha}_{n} \in \tilde{A}_{\tilde{\alpha}_{0}}^{\oplus}$, where " $\exists$ " means "there exists" and " $\forall$ " means "for any", then such $\left\{\tilde{\alpha}_{n}\right\}$ is called an addition sequence derived from $\tilde{\alpha}_{0}$; If $\forall n>N, \tilde{\alpha}_{n} \in \tilde{A}_{\tilde{\alpha}_{0}}^{\ominus}$, then $\left\{\tilde{\alpha}_{n}\right\}$ is a subtraction sequence derived from $\tilde{\alpha}_{0}$.

According to the above concepts, for a fixed IVIFV $\tilde{\alpha}_{0}$, we can always find the unlimited elements of an addition sequence derived from $\tilde{\alpha}_{0}$, which are all contained in $\tilde{A}_{\tilde{\alpha}_{0}}^{\oplus}$ (see Figure 3); and the unlimited elements of a subtraction sequence of $\tilde{\alpha}_{0}$ completely contained in $\tilde{A}_{\tilde{\alpha}_{0}}^{\ominus}$ (see Figure 4):

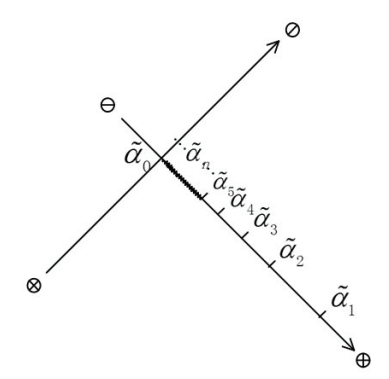

Figure 3. An addition sequence of $\tilde{\alpha}_{0}$

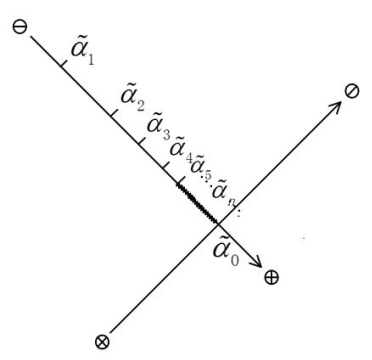

Figure 4. A subtraction sequence of $\tilde{\alpha}_{0}$

Definition 12. Assume that $\left\{\tilde{\alpha}_{n}\right\}(n=1,2, \cdots)$ are a sequence of IVIFVs. If $\exists N \in N^{+}, \forall n>N, \tilde{\alpha}_{n} \in \tilde{A}_{\tilde{\alpha}_{0}}^{\ominus}$, then we call $\left\{\tilde{\alpha}_{n}\right\}$ a division sequence derived from $\tilde{\alpha}_{0}$; If $\forall n>N, \quad \tilde{\alpha}_{n} \in \tilde{A}_{\tilde{\alpha}_{0}}^{\otimes}$, then we call $\left\{\tilde{\alpha}_{n}\right\}$ a multiplication sequence derived from $\tilde{\alpha}_{0}$.

Similarly, from Figure 5, we can see that there are unlimited elements of a division sequence derived from $\tilde{\alpha}_{0}$ which are all in the set $\tilde{A}_{\tilde{\alpha}_{0}}^{\oslash}$. At the same time, from Figure 6, we can see the unlimited elements of a multiplication sequence derived from $\tilde{\alpha}_{0}$ which are all in $\tilde{A}_{\tilde{\alpha}_{0}}^{\otimes}$ :

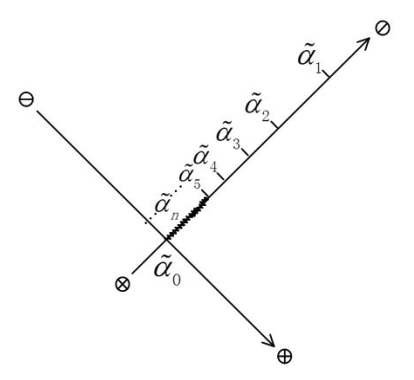

Figure 5. A division sequence of $\tilde{\alpha}_{0}$

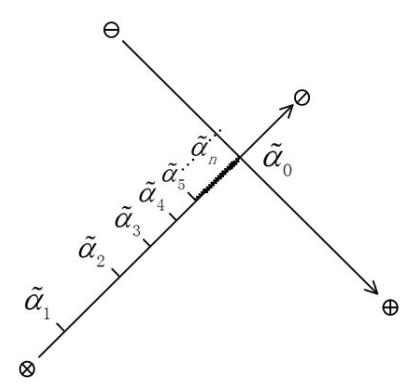

Figure 6. A multiplication sequence of $\tilde{\alpha}_{0}$ 


\subsection{The limits of various sequences of IVIFVs}

In real number field, we use the distance $|b-a|$ of two real numbers $a$ and $b$ to describe their approaching degree. The smaller the distance $|b-a|$ is, the closer the two numbers are. $a$ is infinitely approaching $b$ if and only if the distance $|b-a|$ is infinitely approaching to zero. How to describe the approaching process of two IVIFVs by using their basic operations? For arbitrary IVIFV $\tilde{\alpha}$, because $\tilde{\alpha} \oplus([0,0],[1,1])=\tilde{\alpha}, \tilde{\alpha} \oplus \tilde{\theta} \rightarrow \tilde{\alpha}$ if only both the membership degree interval and the nonmembership degree interval of $\tilde{\theta}$ approach to those of the $\operatorname{IVIFV}([0,0],[1,1])$. Therefore, we define the following limit process $\tilde{\alpha}_{n} \rightarrow \tilde{\alpha}_{0}$ :

Definition 13. For an addition sequence derived from $\tilde{\alpha}_{0}$, denoted by $\left\{\tilde{\alpha}_{n}\right\}(n=1,2, \cdots)$, if $\forall \tilde{\varepsilon}=\left(\left[a_{\tilde{\varepsilon}}, b_{\tilde{\varepsilon}}\right],\left[c_{\tilde{\varepsilon}}, d_{\tilde{\varepsilon}}\right]\right)>_{L}([0,0],[1,1]), \exists N \in N^{+}$, when $\forall n>N$, we have

$$
\tilde{\alpha}_{n} \ominus \tilde{\alpha}_{0}<_{L} \tilde{\varepsilon}
$$

Then we call $\tilde{\alpha}_{0}$ the addition limit of $\left\{\tilde{\alpha}_{n}\right\}$ as $n \rightarrow+\infty$, denoted by $\lim _{n \rightarrow+\infty} \tilde{\alpha}_{n}=\tilde{\alpha}_{0}^{\oplus}$ or $\tilde{\alpha}_{n} \rightarrow \tilde{\alpha}_{0}^{\oplus}$.

Definition 13 shows that when $\tilde{\alpha}_{n} \ominus \tilde{\alpha}_{0} \rightarrow([0,0],[1,1])$, we have $\tilde{\alpha}_{n} \rightarrow \tilde{\alpha}_{0}^{\oplus}$. Similarly, we give the following definition if each element $\tilde{\alpha}_{n}$ of a sequence is contained in $\tilde{A}_{\tilde{\alpha}}^{\ominus}$ :

Definition 14. Let $\left\{\tilde{\alpha}_{n}\right\}$ be a subtraction sequence derived from $\tilde{\alpha}_{0}$. If $\forall \tilde{\varepsilon}=\left(\left[a_{\tilde{\varepsilon}}, b_{\tilde{\varepsilon}}\right],\left[c_{\tilde{\varepsilon}}, d_{\tilde{\varepsilon}}\right]\right)>_{L}([0,0],[1,1]), \exists N \in N^{+}$, when $\forall n>N$, we have

$$
\tilde{\alpha}_{0} \ominus \tilde{\alpha}_{n}<_{L} \tilde{\varepsilon}
$$

then $\tilde{\alpha}_{0}$ is called the subtraction limit of $\left\{\tilde{\alpha}_{n}\right\}$ as $n \rightarrow+\infty$, denoted by $\lim _{n \rightarrow+\infty} \tilde{\alpha}_{n}=\tilde{\alpha}_{0}^{\ominus}$ or $\tilde{\alpha}_{n} \rightarrow \tilde{\alpha}_{0}^{\ominus}$.

We have considered the situations $\tilde{\alpha}_{n} \in \tilde{A}_{\tilde{\alpha}_{0}}^{\oplus}$ and $\tilde{\alpha}_{n} \in \tilde{A}_{\tilde{\alpha}_{0}}^{\ominus}$, and defined the addition and subtraction limits respectively. Next we shall consider the situations when $\quad \tilde{\alpha}_{n} \in \tilde{A}_{\tilde{\alpha}_{0}}^{\otimes} \quad$ and $\quad \tilde{\alpha}_{n} \in \tilde{A}_{\tilde{\alpha}_{0}}^{\ominus} \quad \cdot \quad$ Because $\tilde{\alpha} \otimes([1,1],[0,0])=\tilde{\alpha}, \tilde{\alpha} \otimes \tilde{\theta} \rightarrow \tilde{\alpha}$ so long as both the membership degree interval and the non-membership degree interval of $\tilde{\theta}$ approach to those of $([1,1],[0,0])$, in the following, we shall give the other two limit definitions:

Definition 15. Suppose that $\left\{\tilde{\alpha}_{n}\right\}$ is a division sequence derived from $\quad \tilde{\alpha}_{0}$. If $\forall \tilde{\varepsilon}=\left(\left[a_{\tilde{\varepsilon}}, b_{\tilde{\varepsilon}}\right],\left[c_{\tilde{\varepsilon}}, d_{\tilde{\varepsilon}}\right]\right)$ $<_{L}([1,1],[0,0]), \exists N \in N^{+}$, when $\forall n>N$, we have

$$
\tilde{\alpha}_{0} \oslash \tilde{\alpha}_{n}>_{L} \tilde{\varepsilon}
$$

then $\tilde{\alpha}_{0}$ is the division limit of $\left\{\tilde{\alpha}_{n}\right\}$ as $n \rightarrow+\infty$, denoted by $\lim _{n \rightarrow+\infty} \tilde{\alpha}_{n}=\tilde{\alpha}_{0}^{\oslash}$ or $\tilde{\alpha}_{n} \rightarrow \tilde{\alpha}_{0}^{\oslash}$.

Definition 15 shows that when $\tilde{\alpha}_{0} \oslash \tilde{\alpha}_{n} \rightarrow([1,1],[0,0])$, we have $\tilde{\alpha}_{n} \rightarrow \tilde{\alpha}_{0}^{\oslash}$.

Definition 16. Assume that $\left\{\tilde{\alpha}_{n}\right\}$ is a multiplication sequence derived from $\tilde{\alpha}_{0}$. If $\forall \tilde{\varepsilon}=\left(\left[a_{\tilde{\varepsilon}}, b_{\tilde{\varepsilon}}\right],\left[c_{\tilde{\varepsilon}}, d_{\tilde{\varepsilon}}\right]\right)$ $<_{L}([1,1],[0,0]), \exists N \in N^{+}$, when $\forall n>N$, we have

$$
\tilde{\alpha}_{n} \oslash \tilde{\alpha}_{0}>_{L} \tilde{\varepsilon}
$$

then $\tilde{\alpha}_{0}$ is the multiplication limit of $\left\{\tilde{\alpha}_{n}\right\}$ as $n \rightarrow+\infty$, denoted by $\lim _{n \rightarrow+\infty} \tilde{\alpha}_{n}=\tilde{\alpha}_{0}^{\otimes}$ or $\tilde{\alpha}_{n} \rightarrow \tilde{\alpha}_{0}^{\otimes}$.

Next, we shall give an equivalent characterization of Definition 13. We first make an analysis on Definition 13. Because $\left\{\tilde{\alpha}_{n}\right\}$ is an addition sequence derived from $\tilde{\alpha}_{0}$, we can get

$$
\tilde{\alpha}_{n} \ominus \tilde{\alpha}_{0}=\left(\left[\frac{a_{n}-a_{0}}{1-a_{0}}, \frac{b_{n}-b_{0}}{1-b_{0}}\right],\left[\frac{c_{n}}{c_{0}}, \frac{d_{n}}{d_{0}}\right]\right) \in \Theta
$$

Then Definition 13 shows that for any given $a_{\tilde{\varepsilon}}>0, b_{\tilde{\varepsilon}}>0, c_{\tilde{\varepsilon}}<1, d_{\tilde{\varepsilon}}<1$, we have $\frac{a_{n}-a_{0}}{1-a_{0}}<a_{\tilde{\varepsilon}}$, $\frac{b_{n}-b_{0}}{1-b_{0}}<b_{\tilde{\varepsilon}}$ and $\frac{c_{n}}{c_{0}}>c_{\tilde{\varepsilon}}, \frac{d_{n}}{d_{0}}>d_{\tilde{\varepsilon}}$. Thus by Definition 13, we know $a_{n}-a_{0} \rightarrow 0, b_{n}-b_{0} \rightarrow 0$ and $\frac{c_{n}}{c_{0}} \rightarrow 1, \frac{d_{n}}{d_{0}} \rightarrow 1$ simultaneously. Therefore, we can get the following equivalent characterization of Definition 13:

Theorem 1. Let $\left\{\tilde{\alpha}_{n}\right\}$ be an addition sequence derived from $\tilde{\alpha}_{0}$, with $\tilde{\alpha}_{n}=\left(\left[a_{n}, b_{n}\right],\left[c_{n}, d_{n}\right]\right)$ and $\tilde{\alpha}_{0}=\left(\left[a_{0}, b_{0}\right],\left[c_{0}, d_{0}\right]\right)$, then $\lim _{n \rightarrow+\infty} \tilde{\alpha}_{n}=\tilde{\alpha}_{0}^{\oplus}$ if and only if $\lim _{n \rightarrow+\infty} a_{n}=a_{0}, \lim _{n \rightarrow+\infty} b_{n}=b_{0}$ and $\lim _{n \rightarrow+\infty} c_{n}=c_{0}$, $\lim _{n \rightarrow+\infty} d_{n}=d_{0}$. 
Proof (Sufficiency). Suppose that $\lim _{n \rightarrow+\infty} a_{n}=a_{0}, \lim _{n \rightarrow+\infty} b_{n}=b_{0} \quad$ and $\quad \lim _{n \rightarrow+\infty} c_{n}=c_{0}$, $\lim _{n \rightarrow+\infty} d_{n}=d_{0}$. By the definition of limit in real number field:

(1) $\forall \varepsilon_{1}>0, \exists N_{1} \in N^{+}$, when $\forall n>N_{1}$, we have

$$
a_{n}-a_{0}<\left(1-a_{0}\right) \varepsilon_{1}
$$

(2) $\forall \varepsilon_{2}>0, \exists N_{2} \in N^{+}$, when $\forall n>N_{2}$, we have

$$
b_{n}-b_{0}<\left(1-b_{0}\right) \varepsilon_{2}
$$

(3) $\forall \varepsilon_{3}>0, \exists N_{3} \in N^{+}$, when $\forall n>N_{3}$, we have

$$
1-\frac{c_{n}}{c_{0}}<\varepsilon_{3} \Rightarrow \frac{c_{n}}{c_{0}}>1-\varepsilon_{3}
$$

Similarly, $\forall \varepsilon_{4}>0, \exists N_{4} \in N^{+}$, when $\forall n>N_{4}$, we have

$$
1-\frac{d_{n}}{d_{0}}<\varepsilon_{4} \Rightarrow \frac{d_{n}}{d_{0}}>1-\varepsilon_{4}
$$

Let $N=\max \left(N_{1}, N_{2}, N_{3}, N_{4}\right)$, then when $\forall n>N$, we have $\quad \frac{a_{n}-a_{0}}{1-a_{0}}<\varepsilon_{1}, \frac{b_{n}-b_{0}}{1-b_{0}}<\varepsilon_{2} \quad$ and $\frac{c_{n}}{c_{0}}>1-\varepsilon_{3}, \frac{d_{n}}{d_{0}}>1-\varepsilon_{4}$. In this case, if we take $\tilde{\varepsilon}=\left(\left[\varepsilon_{1}, \varepsilon_{2}\right],\left[1-\varepsilon_{3}, 1-\varepsilon_{4}\right]\right)$, then $\lim _{n \rightarrow+\infty} \tilde{\alpha}_{n}=\tilde{\alpha}_{0}^{\oplus}$.

(Necessity). If $\lim _{n \rightarrow+\infty} \tilde{\alpha}_{n}=\tilde{\alpha}_{0}^{\oplus}$, by Definition 13 , $\forall \tilde{\varepsilon}=\left(\left[a_{\tilde{\varepsilon}}, b_{\tilde{\varepsilon}}\right],\left[c_{\tilde{\varepsilon}}, d_{\tilde{\varepsilon}}\right]\right)>_{L}([0,0],[1,1]), \quad \exists N \in N^{+}$, when $\forall n>N$, we have

That is,

$$
\tilde{\alpha}_{n} \ominus \tilde{\alpha}_{0}<_{L} \tilde{\varepsilon}
$$

$$
\left(\left[\frac{a_{n}-a_{0}}{1-a_{0}}, \frac{b_{n}-b_{0}}{1-b_{0}}\right],\left[\frac{c_{n}}{c_{0}}, \frac{d_{n}}{d_{0}}\right]\right)<_{L}\left(\left[a_{\tilde{\varepsilon}}, b_{\tilde{\varepsilon}}\right],\left[c_{\tilde{\varepsilon}}, d_{\tilde{\varepsilon}}\right]\right)
$$

As $\left\{\tilde{\alpha}_{n}\right\}$ is an addition sequence derived from $\tilde{\alpha}_{0}$, then $\quad 0<\frac{a_{n}-a_{0}}{1-a_{0}}<a_{\tilde{\varepsilon}}, \quad 0<\frac{b_{n}-b_{0}}{1-b_{0}}<b_{\tilde{\varepsilon}} \quad$ and $1>\frac{c_{n}}{c_{0}}>c_{\tilde{\varepsilon}}, 1>\frac{d_{n}}{d_{0}}>d_{\tilde{\varepsilon}}$. By the squeeze theorem of limit in real number field, we can get $\lim _{n \rightarrow+\infty} a_{n}=a_{0}, \lim _{n \rightarrow+\infty} b_{n}=b_{0} \quad$ and $\quad \lim _{n \rightarrow+\infty} c_{n}=c_{0}$, $\lim _{n \rightarrow+\infty} d_{n}=d_{0}$. Thus we complete the proof of Theorem 1.

Furthermore, when $\left\{\tilde{\alpha}_{n}\right\}$ is a subtraction sequence, a division sequence or a multiplication sequence derived from $\tilde{\alpha}_{0}$, we have the similar conclusion.

\section{The continuity and differentiability of IVIFFs}

\subsection{The concept and properties of IVIFFs}

Suppose that $\tilde{\alpha}=([a, b],[c, d])$, and $F$ is a function of IVIFVs, that is,

$$
F(\tilde{\alpha})=\left(\left[f_{1}(a, b, c, d), f_{2}(a, b, c, d)\right],\left[g_{1}(a, b, c, d), g_{2}(a, b, c, d)\right]\right)
$$

If $0 \leq f_{i}(a, b, c, d) \leq 1,0 \leq g_{i}(a, b, c, d) \leq 1, i=1,2$, and $0 \leq f_{2}(a, b, c, d)+g_{2}(a, b, c, d) \leq 1$, then we call the function $F(\tilde{\alpha})$ an interval-valued intuitionistic fuzzy function (IVIFF) of $\tilde{\alpha}$.

For brevity, we denote any given IVIFF

$$
\begin{aligned}
F(\tilde{\alpha})= & \left(\left[f_{1}(a, b, c, d), f_{2}(a, b, c, d)\right],\right. \\
& {\left.\left[g_{1}(a, b, c, d), g_{2}(a, b, c, d)\right]\right) }
\end{aligned}
$$

as $F=\left(\left[f_{1}, f_{2}\right],\left[g_{1}, g_{2}\right]\right)$.

Because $F(\tilde{\alpha}) \ominus F\left(\tilde{\alpha}_{0}\right)$ isn't always an IVIFV, for the purpose of discussing the derivatives of IVIFFs, we want to know in what conditions $F(\tilde{\alpha}) \ominus F\left(\tilde{\alpha}_{0}\right)$ will be an IVIFV. This question relates to the subtraction operation of IVIFV:

Definition 17. Let

$$
\begin{aligned}
& F(\tilde{\alpha})=\left(\left[f_{1}(a, b, c, d), f_{2}(a, b, c, d)\right],\right. \\
& \left.\left[g_{1}(a, b, c, d), g_{2}(a, b, c, d)\right]\right) \text { be an IVIFF of } \\
& \tilde{\alpha}=([a, b],[c, d]) \text {, then we denote } \\
& \tilde{S}^{\oplus}\left(\tilde{\alpha}_{0}, F\right)=\left\{\tilde{\alpha} \mid \tilde{\alpha} \in \tilde{A}_{\tilde{\alpha}_{0}}^{\oplus},\right. \\
& 0 \leq \frac{g_{1}(a, b, c, d)}{g_{1}\left(a_{0}, b_{0}, c_{0}, d_{0}\right)} \leq \frac{1-f_{1}(a, b, c, d)}{1-f_{1}\left(a_{0}, b_{0}, c_{0}, d_{0}\right)} \leq 1, \\
& \left.0 \leq \frac{g_{2}(a, b, c, d)}{g_{2}\left(a_{0}, b_{0}, c_{0}, d_{0}\right)} \leq \frac{1-f_{2}(a, b, c, d)}{1-f_{2}\left(a_{0}, b_{0}, c_{0}, d_{0}\right)} \leq 1\right\}
\end{aligned}
$$

as the addition area of $F(\tilde{\alpha})$ at $\tilde{\alpha}_{0}$.

Using the subtraction operation of IVIFV, we can get

$F(\tilde{\alpha}) \ominus F\left(\tilde{\alpha}_{0}\right)=\left(\left[f_{1}(a, b, c, d), f_{2}(a, b, c, d)\right],\left[g_{1}(a, b, c, d), g_{2}(a, b, c, d)\right]\right)$ $\ominus\left(\left[f_{1}\left(a_{0}, b_{0}, c_{0}, d_{0}\right), f_{2}\left(a_{0}, b_{0}, c_{0}, d_{0}\right)\right],\left[g_{1}\left(a_{0}, b_{0}, c_{0}, d_{0}\right), g_{2}\left(a_{0}, b_{0}, c_{0}, d_{0}\right)\right]\right)$ 


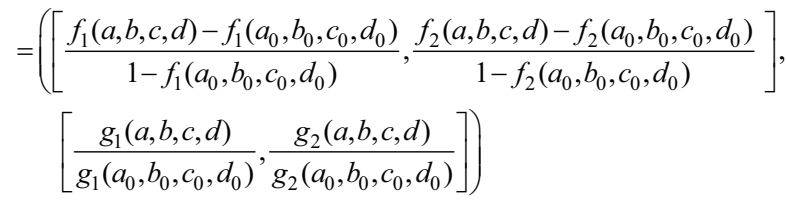

From the analysis above, we know that $\forall \tilde{\alpha} \in \tilde{S}^{\oplus}\left(\tilde{\alpha}_{0}, F\right), F(\tilde{\alpha}) \ominus F\left(\tilde{\alpha}_{0}\right)$ is still an IVIFV when $\tilde{\alpha} \odot \tilde{\alpha}_{0}$ is an IVIFV.

\section{Definition $18 . \quad$ Suppose that} $F(\tilde{\alpha})=\left(\left[f_{1}(a, b, c, d), f_{2}(a, b, c, d)\right]\right.$, $\left.\left[g_{1}(a, b, c, d), g_{2}(a, b, c, d)\right]\right)$

$\tilde{\alpha}=([a, b],[c, d])$, then we denote

$\tilde{S}^{\ominus}\left(\tilde{\alpha}_{0}, F\right)=\left\{\tilde{\alpha} \mid \tilde{\alpha} \in \tilde{A}_{\tilde{\alpha}_{0}}^{\ominus}, 0 \leq \frac{g_{1}\left(a_{0}, b_{0}, c_{0}, d_{0}\right)}{g_{1}(a, b, c, d)} \leq \frac{1-f_{1}\left(a_{0}, b_{0}, c_{0}, d_{0}\right)}{1-f_{1}(a, b, c, d)} \leq 1\right.$, $\left.0 \leq \frac{g_{2}\left(a_{0}, b_{0}, c_{0}, d_{0}\right)}{g_{2}(a, b, c, d)} \leq \frac{1-f_{2}\left(a_{0}, b_{0}, c_{0}, d_{0}\right)}{1-f_{2}(a, b, c, d)} \leq 1\right\}$

as the subtraction area of $F(\tilde{\alpha})$ at $\tilde{\alpha}_{0}$.

By Definition 18, we know that $\forall \tilde{\alpha} \in \tilde{S}^{\ominus}\left(\tilde{\alpha}_{0}, F\right)$, $F\left(\tilde{\alpha}_{0}\right) \ominus F(\tilde{\alpha})$ is still an IVIFV when $\tilde{\alpha}_{0} \ominus \tilde{\alpha}$ is an IVIFV.

Like the addition and subtraction areas of $F(\tilde{\alpha})$ at $\tilde{\alpha}_{0}$, we can define the corresponding division and multiplication areas:

Definition 19. Assume that $\begin{aligned} F(\tilde{\alpha})= & \left(\left[f_{1}(a, b, c, d), f_{2}(a, b, c, d)\right], \quad \text { is an IVIFF of }\right. \\ & {\left.\left[g_{1}(a, b, c, d), g_{2}(a, b, c, d)\right]\right) }\end{aligned}$ $\tilde{\alpha}=([a, b],[c, d])$, then we denote $\tilde{S}^{\ominus}\left(\tilde{\alpha}_{0}, F\right)=\left\{\tilde{\alpha} \mid \tilde{\alpha} \in \tilde{A}_{\tilde{\alpha}_{0}}^{\ominus}\right.$, $0 \leq \frac{f_{1}\left(a_{0}, b_{0}, c_{0}, d_{0}\right)}{f_{1}(a, b, c, d)} \leq \frac{1-g_{1}\left(a_{0}, b_{0}, c_{0}, d_{0}\right)}{1-g_{1}(a, b, c, d)} \leq 1$, $\left.0 \leq \frac{f_{2}\left(a_{0}, b_{0}, c_{0}, d_{0}\right)}{f_{2}(a, b, c, d)} \leq \frac{1-g_{2}\left(a_{0}, b_{0}, c_{0}, d_{0}\right)}{1-g_{2}(a, b, c, d)} \leq 1\right\}$ as the division area of $F(\tilde{\alpha})$ at $\tilde{\alpha}_{0}$.

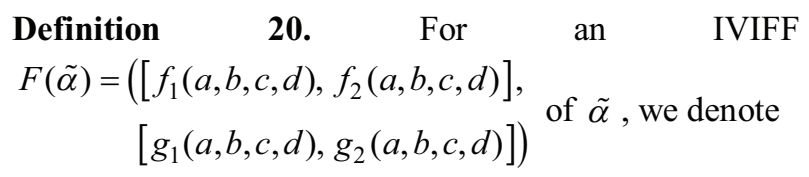

$$
\left.\begin{array}{l}
\tilde{S}^{\otimes}\left(\tilde{\alpha}_{0}, F\right)=\left\{\tilde{\alpha} \mid \tilde{\alpha} \in \tilde{A}_{\tilde{\alpha}_{0}}^{\otimes},\right. \\
0 \leq \frac{f_{1}(a, b, c, d)}{f_{1}\left(a_{0}, b_{0}, c_{0}, d_{0}\right)} \leq \frac{1-g_{1}(a, b, c, d)}{1-g_{1}\left(a_{0}, b_{0}, c_{0}, d_{0}\right)} \leq 1, \\
0 \leq \frac{f_{2}(a, b, c, d)}{f_{2}\left(a_{0}, b_{0}, c_{0}, d_{0}\right)} \leq \frac{1-g_{2}(a, b, c, d)}{1-g_{2}\left(a_{0}, b_{0}, c_{0}, d_{0}\right)} \leq 1
\end{array}\right\}
$$

as the multiplication area of $F(\tilde{\alpha})$ at $\tilde{\alpha}_{0}$.

From Definitions 19 and 20, we know that $\forall \tilde{\alpha} \in \tilde{S}^{\ominus}\left(\tilde{\alpha}_{0}, F\right), F\left(\tilde{\alpha}_{0}\right) \oslash F(\tilde{\alpha})$ is still an IFV when $\tilde{\alpha}_{0} \ominus \tilde{\alpha} \quad$ is an IVIFV, and $\forall \tilde{\alpha} \in \tilde{S}^{\otimes}\left(\tilde{\alpha}_{0}, F\right)$, $F\left(\tilde{\alpha}_{0}\right) \oslash F(\tilde{\alpha})$ is still an IVIFV when $\tilde{\alpha}_{0} \ominus \tilde{\alpha}$ is an IVIFV.

In the following, we shall investigate the relationships of the previous results by some simple examples:

(1) If $F(\tilde{\alpha})=\tilde{\alpha}$, then $f_{1}(a, b, c, d)=a, f_{2}(a, b, c, d)=b$ and $g_{1}(a, b, c, d)=c, g_{2}(a, b, c, d)=d$. If $\forall \tilde{\alpha} \in \tilde{A}_{\tilde{\alpha}_{0}}^{\oplus}$, then we can get $0 \leq \frac{c}{c_{0}} \leq \frac{1-a}{1-a_{0}} \leq 1$ and $0 \leq \frac{d}{d_{0}} \leq \frac{1-b}{1-b_{0}} \leq 1$. So $\forall \tilde{\alpha} \in \tilde{A}_{\tilde{\alpha}_{0}}^{\oplus}$, we have

$$
0 \leq \frac{g_{1}(a, b, c, d)}{g_{1}\left(a_{0}, b_{0}, c_{0}, d_{0}\right)} \leq \frac{1-f_{1}(a, b, c, d)}{1-f_{1}\left(a_{0}, b_{0}, c_{0}, d_{0}\right)} \leq 1
$$

and

$$
0 \leq \frac{g_{2}(a, b, c, d)}{g_{2}\left(a_{0}, b_{0}, c_{0}, d_{0}\right)} \leq \frac{1-f_{2}(a, b, c, d)}{1-f_{2}\left(a_{0}, b_{0}, c_{0}, d_{0}\right)} \leq 1
$$

that is, $\tilde{S}^{\oplus}\left(\tilde{\alpha}_{0}, F\right)=\tilde{A}_{\tilde{\alpha}_{0}}^{\oplus}$. Similarly, we can also get

$$
\tilde{S}^{\ominus}\left(\tilde{\alpha}_{0}, F\right)=\tilde{A}_{\tilde{\alpha}_{0}}^{\ominus}, \tilde{S}^{\ominus}\left(\tilde{\alpha}_{0}, F\right)=\tilde{A}_{\tilde{\alpha}_{0}}^{\ominus}, \tilde{S}^{\otimes}\left(\tilde{\alpha}_{0}, F\right)=\tilde{A}_{\tilde{\alpha}_{0}}^{\otimes}
$$

(2) If

$$
F(\tilde{X})=\tilde{X} \oplus \tilde{\alpha}_{1}=\left(\left[a+a_{1}-a a_{1}, b+b_{1}-b b_{1}\right],\left[c c_{1}, d d_{1}\right]\right)
$$
where $\tilde{X}=([a, b],[c, d])$ and $\tilde{\alpha}_{1}=\left(\left[a_{1}, b_{1}\right],\left[c_{1}, d_{1}\right]\right)$. Let

and

$$
\begin{aligned}
& f_{1}(a, b, c, d)=a+a_{1}-a a_{1} \\
& f_{2}(a, b, c, d)=b+b_{1}-b b_{1}
\end{aligned}
$$

$$
g_{1}(a, b, c, d)=c c_{1}, g_{2}(a, b, c, d)=d d_{1}
$$

For any $\tilde{\alpha} \in \tilde{A}_{\tilde{\alpha}_{0}}^{\oplus}$, we have $0 \leq \frac{c}{c_{0}} \leq \frac{1-a}{1-a_{0}} \leq 1$ and $0 \leq \frac{d}{d_{0}} \leq \frac{1-b}{1-b_{0}} \leq 1$, which are equivalent to 


$$
0 \leq \frac{c c_{1}}{c_{0} c_{1}} \leq \frac{1-\left(a+a_{1}-a a_{1}\right)}{1-\left(a_{0}+a_{1}-a_{0} a_{1}\right)} \leq 1
$$

and

$$
0 \leq \frac{d d_{1}}{d_{0} d_{1}} \leq \frac{1-\left(b+b_{1}-b b_{1}\right)}{1-\left(b_{0}+b_{1}-b_{0} b_{1}\right)} \leq 1
$$

respectively, which means that the following inequalities hold:

$$
\begin{aligned}
& 0 \leq \frac{g_{1}(a, b, c, d)}{g_{1}\left(a_{0}, b_{0}, c_{0}, d_{0}\right)} \leq \frac{1-f_{1}(a, b, c, d)}{1-f_{1}\left(a_{0}, b_{0}, c_{0}, d_{0}\right)} \leq 1 \\
& 0 \leq \frac{g_{2}(a, b, c, d)}{g_{2}\left(a_{0}, b_{0}, c_{0}, d_{0}\right)} \leq \frac{1-f_{2}(a, b, c, d)}{1-f_{2}\left(a_{0}, b_{0}, c_{0}, d_{0}\right)} \leq 1
\end{aligned}
$$

Therefore, $\quad \tilde{S}^{\oplus}\left(\tilde{\alpha}_{0}, F\right)=\tilde{A}_{\tilde{\alpha}_{0}}^{\oplus}$. Similarly, we have $\tilde{S}^{\ominus}\left(\tilde{\alpha}_{0}, F\right)=\tilde{A}_{\tilde{\alpha}_{0}}^{\ominus}$.

(3) For the case that

$\operatorname{IIFWA}_{w}\left(\tilde{\alpha}_{1}, \tilde{\alpha}_{2}, \ldots \tilde{\alpha}_{n}\right)=$

$$
\left(\left[1-\prod_{j=1}^{n}\left(1-a_{j}\right)^{w_{j}}, 1-\prod_{j=1}^{n}\left(1-b_{j}\right)^{w_{j}}\right],\left[\prod_{j=1}^{n} c_{j}^{w_{j}}, \prod_{j=1}^{n} d_{j}^{w_{j}}\right]\right)
$$

where $\quad \tilde{\alpha}_{j}=\left(\left[a_{j}, b_{j}\right],\left[c_{j}, d_{j}\right]\right), j=1,2, \ldots, n \quad . \quad$ For $\forall \tilde{\alpha}_{j}^{\prime} \in \tilde{A}_{\tilde{\alpha}_{j}}^{\oplus}$, with $\tilde{\alpha}_{j}^{\prime}=\left(\left[a_{j}^{\prime}, b_{j}^{\prime}\right],\left[c_{j}^{\prime}, d_{j}^{\prime}\right]\right)$, we can get

$$
\begin{aligned}
& \frac{c_{j}^{\prime}}{c_{j}} \leq \frac{\left(1-a_{j}^{\prime}\right)}{\left(1-a_{j}\right)} \Leftrightarrow\left(\frac{c_{j}^{\prime}}{c_{j}}\right)^{w_{j}} \leq\left(\frac{1-a_{j}^{\prime}}{1-a_{j}}\right)^{w_{j}} \Leftrightarrow \\
& \frac{\left(c_{j}^{\prime}\right)^{w_{j}} \prod_{\substack{i=1 \\
i \neq j}}^{n} c_{i}^{w_{i}}}{c_{j}^{w_{j}} \prod_{\substack{i=1 \\
i \neq j}}^{n} c_{i}^{w_{i}}} \leq \frac{\left(1-a_{j}^{\prime}\right)^{w_{j}} \prod_{\substack{i=1 \\
i \neq j}}^{n}\left(1-a_{i}\right)^{w_{i}}}{\left(1-a_{j}\right)^{w_{j}} \prod_{\substack{i=1 \\
i \neq j}}^{n}\left(1-a_{i}\right)^{w_{i}}}
\end{aligned}
$$

and

$$
\begin{aligned}
& \frac{d_{j}^{\prime}}{d_{j}} \leq \frac{\left(1-b_{j}^{\prime}\right)}{\left(1-b_{j}\right)} \Leftrightarrow\left(\frac{d_{j}^{\prime}}{d_{j}}\right)^{w_{j}} \leq\left(\frac{1-b_{j}^{\prime}}{1-b_{j}}\right)^{w_{j}} \Leftrightarrow \\
& \frac{\left(d_{j}^{\prime}\right)^{w_{j}} \prod_{\substack{i=1 \\
i \neq j}}^{n} d_{i}^{w_{i}}}{d_{j}^{w_{j}} \prod_{\substack{i=1 \\
i \neq j}}^{n} d_{i}^{w_{i}}} \leq \frac{\left(1-b_{j}^{\prime}\right)^{w_{j}} \prod_{\substack{i=1 \\
i \neq j}}^{n}\left(1-b_{i}\right)^{w_{i}}}{\left(1-b_{j}\right)^{w_{j}} \prod_{\substack{i=1 \\
i \neq j}}^{n}\left(1-b_{i}\right)^{w_{i}}}
\end{aligned}
$$

Then we get $\tilde{S}^{\oplus}\left(\tilde{\alpha}_{j}, I I F W A\right)=\tilde{A}_{\tilde{\alpha}_{j}}^{\oplus}$. Similarly, we have $\tilde{S}^{\ominus}\left(\tilde{\alpha}_{j}, I I F W A\right)=\tilde{A}_{\tilde{\alpha}_{j}}^{\ominus}$.
(4) For the case that

$$
\operatorname{IIFWG}_{w}\left(\tilde{\alpha}_{1}, \tilde{\alpha}_{2}, \ldots \tilde{\alpha}_{n}\right)=
$$

$$
\left(\left[\prod_{j=1}^{n} a_{j}^{w_{j}}, \prod_{j=1}^{n} b_{j}^{w_{j}}\right],\left[1-\prod_{j=1}^{n}\left(1-c_{j}\right)^{w_{j}}, 1-\prod_{j=1}^{n}\left(1-d_{j}\right)^{w_{j}}\right]\right)
$$

where $\quad \tilde{\alpha}_{j}=\left(\left[a_{j}, b_{j}\right],\left[c_{j}, d_{j}\right]\right), j=1,2, \ldots, n \quad . \quad$ For $\forall \tilde{\alpha}_{j}^{\prime} \in \tilde{A}_{\tilde{\alpha}_{j}}^{\ominus}$, with $\tilde{\alpha}_{j}^{\prime}=\left(\left[a_{j}^{\prime}, b_{j}^{\prime}\right],\left[c_{j}^{\prime}, d_{j}^{\prime}\right]\right)$, it follows that

$$
\begin{aligned}
& \frac{a_{j}}{a_{j}^{\prime}} \leq \frac{1-c_{j}}{1-c_{j}^{\prime}} \Leftrightarrow\left(\frac{a_{j}}{a_{j}^{\prime}}\right)^{w_{j}} \leq\left(\frac{1-c_{j}}{1-c_{j}^{\prime}}\right)^{w_{j}} \Leftrightarrow \\
& \frac{\left(a_{j}\right)^{w_{j}} \prod_{\substack{i=1 \\
i \neq j}}^{n} a_{i}^{w_{i}}}{\left(a_{j}^{\prime}\right)^{w_{j}} \prod_{\substack{i=1 \\
i \neq j}}^{n} a_{i}^{w_{i}}} \leq \frac{\left(1-c_{j}\right)^{w_{j}} \prod_{\substack{i=1 \\
i \neq j}}^{n}\left(1-c_{i}\right)^{w_{i}}}{\left(1-c_{j}^{\prime}\right)^{w_{j}} \prod_{\substack{i=1 \\
i \neq j}}^{n}\left(1-c_{i}\right)^{w_{i}}}
\end{aligned}
$$

and

$$
\begin{aligned}
& \frac{b_{j}}{b_{j}^{\prime}} \leq \frac{1-d_{j}}{1-d_{j}^{\prime}} \Leftrightarrow\left(\frac{b_{j}}{b_{j}^{\prime}}\right)^{w_{j}} \leq\left(\frac{1-d_{j}}{1-d_{j}^{\prime}}\right)^{w_{j}} \Leftrightarrow \\
& \frac{\left(b_{j}\right)^{w_{j}} \prod_{\substack{i=1 \\
i \neq j}}^{n} b_{i}^{w_{i}}}{\left(b_{j}^{\prime}\right)^{w_{j}} \prod_{\substack{i=1 \\
i \neq j}}^{n} b_{i}^{w_{i}}} \leq \frac{\left(1-d_{j}\right)^{w_{j}} \prod_{\substack{i=1 \\
i \neq j}}^{n}\left(1-d_{i}\right)^{w_{i}}}{\left(1-d_{j}^{\prime}\right)^{w_{j}} \prod_{\substack{i=1 \\
i \neq j}}^{n}\left(1-d_{i}\right)^{w_{i}}}
\end{aligned}
$$

then $\tilde{S}^{\ominus}\left(\tilde{\alpha}_{j}, I I F W G\right)=\tilde{A}_{\tilde{\alpha}_{j}}^{\ominus}$. We can also obtain $\tilde{S}^{\otimes}\left(\tilde{\alpha}_{j}, I I F W G\right)=\tilde{A}_{\tilde{\alpha}_{j}}^{\otimes}$ in a similar way.

\subsection{The continuity of IVIFF}

In real number field, the continuity of a real function $f(x)$ is a very important property. If $f(x)-f\left(x_{0}\right) \rightarrow 0$ when $x \rightarrow x_{0}$, then the function $f(x)$ is continuous. After defining the convergence of sequences of IVIFVs, it is natural for us to ask what the continuity of an IVIFF is. In the following, we shall focus on this issue:

Definition 21. Let $F(\tilde{X})$ be an IVIFF of $\tilde{X}$. If $\forall \tilde{\varepsilon}=\left(\left[a_{\tilde{\varepsilon}}, b_{\tilde{\varepsilon}}\right],\left[c_{\tilde{\varepsilon}}, d_{\tilde{\varepsilon}}\right]\right)>_{L}([0,0],[1,1])$ $\exists \tilde{\delta}=\left(\left[a_{\delta}, b_{\delta}\right],\left[c_{\delta}, d_{\delta}\right]\right)$, for any $\tilde{X} \in \tilde{S}^{\oplus}\left(\tilde{X}_{0}, F\right)$, when $([0,0],[1,1])<_{L} \tilde{X} \ominus \tilde{X}_{0}<_{L} \tilde{\delta} \quad, \quad$ there is $F(\tilde{X}) \ominus F\left(\tilde{X}_{0}\right)<_{L} \tilde{\varepsilon}$, then $F(\tilde{X})$ is continuous at $\tilde{X}_{0}$ 
in the addition area, denoted by $\lim _{\tilde{X} \rightarrow \tilde{X}_{0}^{\oplus}} F(\tilde{X})=F\left(\tilde{X}_{0}\right)$.

Definition 21 shows that if $F(\tilde{X})$ is continuous at $\tilde{X}_{0}$ in the addition area $\tilde{S}^{\oplus}\left(\tilde{X}_{0}, F\right)$, then when $\tilde{X} \odot \tilde{X}_{0} \rightarrow([0,0],[1,1]) \quad$, we have $F(\tilde{X}) \ominus F\left(\tilde{X}_{0}\right) \rightarrow([0,0],[1,1])$.

Definition 22. Suppose that $F(\tilde{X})$ is an IVIFF of $\tilde{X}$. If $\quad \forall \tilde{\varepsilon}=\left(\left[a_{\tilde{\varepsilon}}, b_{\tilde{\varepsilon}}\right],\left[c_{\tilde{\varepsilon}}, d_{\tilde{\varepsilon}}\right]\right)>_{L}([0,0],[1,1]) \quad$ ， $\exists \tilde{\delta}=\left(\left[a_{\delta}, b_{\delta}\right],\left[c_{\delta}, d_{\delta}\right]\right)$, making $\forall \tilde{X} \in \tilde{S}^{\ominus}\left(\tilde{X}_{0}, F\right)$ and $([0,0],[1,1])<_{L} \tilde{X}_{0} \ominus \tilde{X}<_{L} \tilde{\delta} \quad, \quad$ such that $F\left(\tilde{X}_{0}\right) \ominus F(\tilde{X})<_{L} \tilde{\varepsilon}$. Then $F(\tilde{X})$ is continuous at $\tilde{X}_{0}$ in the subtraction area, denoted by $\lim _{\tilde{X} \rightarrow \tilde{X}_{0}^{\ominus}} F(\tilde{X})=F\left(\tilde{X}_{0}\right)$.

Definition 23. Assume that $F(\tilde{X})$ is an IVIFF of $\tilde{X}$. If $\forall \tilde{\varepsilon}=\left(\left[a_{\tilde{\varepsilon}}, b_{\tilde{\varepsilon}}\right],\left[c_{\tilde{\varepsilon}}, d_{\tilde{\varepsilon}}\right]\right)<_{L}([1,1],[0,0])$ $\exists \tilde{\delta}=\left(\left[a_{\delta}, b_{\delta}\right],\left[c_{\delta}, d_{\delta}\right]\right)$, such that $\tilde{X} \in \tilde{S}^{\ominus}\left(\tilde{X}_{0}, F\right)$ and $\tilde{\delta}<_{L} \tilde{X}_{0} \oslash \tilde{X}<_{L}([1,1],[0,0]) \quad$ satisfy $F\left(\tilde{X}_{0}\right) \oslash F(\tilde{X})>_{L} \tilde{\varepsilon}$, then $F(\tilde{X})$ is continuous at $\tilde{X}_{0}$ in the division area, denoted by $\lim _{\tilde{X} \rightarrow \tilde{X}_{0}^{\odot}} F(\tilde{X})=F\left(\tilde{X}_{0}\right)$.

Definition 24. For a given IVIFF $F(\tilde{X})$ of $\tilde{X}$, if $\forall \tilde{\varepsilon}=\left(\left[a_{\tilde{\varepsilon}}, b_{\tilde{\varepsilon}}\right],\left[c_{\tilde{\varepsilon}}, d_{\tilde{\varepsilon}}\right]\right)<_{L}([1,1],[0,0])$ $\exists \tilde{\delta}=\left(\left[a_{\delta}, b_{\delta}\right],\left[c_{\delta}, d_{\delta}\right]\right)$, for arbitrary $\tilde{X} \in \tilde{S}^{\otimes}\left(\tilde{X}_{0}, F\right)$ when $\tilde{\delta}<_{L} \tilde{X} \oslash \tilde{X}_{0}<_{L}([1,1],[0,0])$, the inequality $F(\tilde{X}) \oslash F\left(\tilde{X}_{0}\right)>_{L} \tilde{\varepsilon}$ holds, then $F(\tilde{X})$ is continuous at $\tilde{X}_{0}$ in the multiplication area, denoted by $\lim _{\tilde{X} \rightarrow \tilde{X}_{0}^{\otimes}} F(\tilde{X})=F\left(\tilde{X}_{0}\right)$.

\subsection{The differentiability of IVIFFs}

As we all know, in the real number field, the derivativeness of a real function $f(x)$ at a real number $x$ depends on the existence of the following limit:

$$
\lim _{x^{\prime} \rightarrow x} \frac{f\left(x^{\prime}\right)-f(x)}{x^{\prime}-x}
$$

If $f\left(x^{\prime}\right)-f(x) \nrightarrow 0$, then the above limit might be equal to the worthless infinity. But, if $f(x)$ is continuous, then $\lim _{x^{\prime} \rightarrow x} \frac{f\left(x^{\prime}\right)-f(x)}{x^{\prime}-x}$ might be equal to a constant, and then we can gain the derivative of the function $f(x)$ at a real number $x$ :

$$
\frac{d f(x)}{d x}=\lim _{x^{\prime} \rightarrow x} \frac{f\left(x^{\prime}\right)-f(x)}{x^{\prime}-x} .
$$

Under interval-valued intuitionistic fuzzy environment, we try to use the idea of gaining the derivative of a real function to define the derivative of an IVIFF. We first analyze the value of $\lim _{\tilde{\alpha}^{\prime} \rightarrow \tilde{\alpha}^{\oplus}} \frac{F\left(\tilde{\alpha}^{\prime}\right) \ominus F(\tilde{\alpha})}{\tilde{\alpha}^{\prime} \ominus \tilde{\alpha}}$. From Definition 13, we know that $\quad \tilde{\alpha}^{\prime} \ominus \tilde{\alpha} \rightarrow([0,0],[1,1]) \quad$ when $\quad \tilde{\alpha}^{\prime} \rightarrow \tilde{\alpha}^{\oplus}$. Then based on the subtraction operation of IVIFVs introduced in Section 2, on the one hand, if $F(\tilde{\alpha}) \ominus F(\tilde{\alpha}) \nrightarrow([0,0],[1,1])$, then the value $\lim _{\tilde{\alpha}^{\prime} \rightarrow \tilde{\alpha}^{\oplus}} \frac{F\left(\tilde{\alpha}^{\prime}\right) \ominus F(\tilde{\alpha})}{\tilde{\alpha}^{\prime} \ominus \tilde{\alpha}} \quad$ is equal to the IVIFV $([0,0],[1,1])$ that takes no information about the divisor and the dividend, and is given only for satisfying the closure of division. On the other hand, so long as the function $F(\tilde{\alpha})$ is continuous, the value $F\left(\tilde{\alpha}^{\prime}\right) \ominus F(\tilde{\alpha})$ will approach $([0,0],[1,1]) \quad$ when $\tilde{\alpha}^{\prime} \ominus \tilde{\alpha} \rightarrow([0,0],[1,1])$. So in this case we can catch the value $\lim _{\tilde{\alpha}^{\prime} \rightarrow \tilde{\alpha}^{\oplus}} \frac{F\left(\tilde{\alpha}^{\prime}\right) \ominus F(\tilde{\alpha})}{\tilde{\alpha}^{\prime} \ominus \tilde{\alpha}}$, which would not always equal to the IVIFV $([0,0],[1,1])$.

After the above analysis, we first give the derivative of an IVIFF at an IVIFV $\tilde{\alpha}$ in the addition direction:

Definition 25. If $\lim _{\tilde{\alpha}^{\prime} \rightarrow \tilde{\alpha}^{\oplus}} \frac{F\left(\tilde{\alpha}^{\prime}\right) \ominus F(\tilde{\alpha})}{\tilde{\alpha}^{\prime} \ominus \tilde{\alpha}}$ is an IVIFV, then the function $F(\tilde{\alpha})$ is called to be differentiable at $\tilde{\alpha}$ in the addition direction, and the limit value is called the derivative of $F(\tilde{\alpha})$ at $\tilde{\alpha}$.

Then we give the following necessary and sufficient condition for the differentiability of an IVIFF $F(\tilde{\alpha})$ :

Theorem 2. Let 
$\begin{aligned} F(\tilde{\alpha})= & \left(\left[f_{1}(a, b, c, d), f_{2}(a, b, c, d)\right], \quad \text { be an IVIFF of }\right. \\ & {\left.\left[g_{1}(a, b, c, d), g_{2}(a, b, c, d)\right]\right) }\end{aligned}$ $\tilde{\alpha}=([a, b],[c, d])$, then $F(\tilde{\alpha})$ is differentiable in the addition direction of $\tilde{\alpha}$, if and only if $\left\{\begin{array}{l}\frac{\partial f_{1}(a, b, c, d)}{\partial b}=\frac{\partial f_{1}(a, b, c, d)}{\partial c}=\frac{\partial f_{1}(a, b, c, d)}{\partial d}=0 \\ \frac{\partial f_{2}(a, b, c, d)}{\partial a}=\frac{\partial f_{2}(a, b, c, d)}{\partial c}=\frac{\partial f_{2}(a, b, c, d)}{\partial d}=0 \\ \frac{\partial g_{1}(a, b, c, d)}{\partial a}=\frac{\partial g_{1}(a, b, c, d)}{\partial b}=\frac{\partial g_{1}(a, b, c, d)}{\partial d}=0 \\ \frac{\partial g_{2}(a, b, c, d)}{\partial a}=\frac{\partial g_{2}(a, b, c, d)}{\partial b}=\frac{\partial g_{2}(a, b, c, d)}{\partial c}=0\end{array}\right.$

and

$$
\left\{\begin{array}{c}
0 \leq \frac{1-a}{1-f_{1}(a, b, c, d)} \frac{\partial f_{1}(a, b, c, d)}{\partial a} \leq 1 \\
0 \leq \frac{c}{g_{1}(a, b, c, d)} \frac{\partial g_{1}(a, b, c, d)}{\partial c} \leq 1 \\
0 \leq \frac{1-b}{1-f_{2}(a, b, c, d)} \frac{\partial f_{2}(a, b, c, d)}{\partial b} \leq \frac{d}{g_{2}(a, b, c, d)} \frac{\partial g_{2}(a, b, c, d)}{\partial d} \leq 1
\end{array}\right.
$$

Under the above sufficient and necessary condition, and $F(\tilde{\alpha})=\left(\left[f_{1}(a), f_{2}(b)\right],\left[g_{1}(c), g_{2}(d)\right]\right)$, the derivative $F(\tilde{\alpha})$ at $\tilde{\alpha}$ can be calculated as follows:

$$
\begin{array}{r}
\frac{d F(\tilde{\alpha})}{d \tilde{\alpha}}=\left(\left[\frac{1-a}{1-f_{1}(a)} f_{1}^{\prime}(a), \frac{1-b}{1-f_{2}(b)} f_{2}^{\prime}(b)\right],\right. \\
\left.\left[1-\frac{c}{g_{1}(c)} g_{1}^{\prime}(c), 1-\frac{d}{g_{2}(d)} g_{2}^{\prime}(d)\right]\right)
\end{array}
$$

Proof. Let

$$
\begin{aligned}
\tilde{\alpha}^{\prime}= & ([a+\Delta a, b+\Delta b],[c+\Delta c, d+\Delta d])= \\
& \left(\left[a^{\prime}, b^{\prime}\right],\left[c^{\prime}, d^{\prime}\right]\right) \in \tilde{S}^{\oplus}(\tilde{\alpha}, F)
\end{aligned}
$$

then

$$
\begin{aligned}
F\left(\tilde{\alpha}^{\prime}\right)= & \left(\left[f_{1}\left(a^{\prime}, b^{\prime}, c^{\prime}, d^{\prime}\right), f_{2}\left(a^{\prime}, b^{\prime}, c^{\prime}, d^{\prime}\right)\right],\right. \\
& {\left.\left[g_{1}\left(a^{\prime}, b^{\prime}, c^{\prime}, d^{\prime}\right), g_{2}\left(a^{\prime}, b^{\prime}, c^{\prime}, d^{\prime}\right)\right]\right) }
\end{aligned}
$$

For brevity, we use the following notations in the proof process:

Thus,

$$
\left(I^{\prime}\right)=\left(a^{\prime}, b^{\prime}, c^{\prime}, d^{\prime}\right),(I)=(a, b, c, d)
$$

$$
\begin{aligned}
& \frac{d F(\tilde{\alpha})}{d \tilde{\alpha}}=\lim _{\tilde{\alpha}^{\prime} \rightarrow \tilde{\alpha}^{\oplus}} \frac{F\left(\tilde{\alpha}^{\prime}\right) \ominus F(\tilde{\alpha})}{\tilde{\alpha}^{\prime} \ominus \tilde{\alpha}} \\
& =\lim _{\substack{\alpha \rightarrow a \\
b \rightarrow b \\
b \rightarrow b}} \frac{\left(\left[f_{1}\left(I^{\prime}\right), f_{2}\left(I^{\prime}\right)\right],\left[g_{1}\left(I^{\prime}\right), g_{2}\left(I^{\prime}\right)\right]\right) \ominus\left(\left[f_{1}(I), f_{2}(I)\right],\left[g_{1}(I), g_{2}(I)\right]\right)}{\left(\left[a^{\prime}, b^{\prime}\right],\left[c^{\prime}, d^{\prime}\right]\right) \ominus([a, b],[c, d])} \\
& \begin{array}{l}
c^{\prime} \rightarrow c \\
d^{\prime} \rightarrow d
\end{array} \\
& =\lim _{\substack{a^{\prime} \rightarrow a \\
b \rightarrow b \\
c^{\prime} \rightarrow c \\
d^{\prime} \rightarrow d}} \frac{\left(\left[\frac{f_{1}\left(I^{\prime}\right)-f_{1}(I)}{1-f_{1}(I)}, \frac{f_{2}\left(I^{\prime}\right)-f_{2}(I)}{1-f_{2}(I)}\right],\left[\frac{g_{1}\left(I^{\prime}\right)}{g_{1}(I)}, \frac{g_{2}\left(I^{\prime}\right)}{g_{2}(I)}\right]\right)}{\left(\left[\frac{a^{\prime}-a}{1-a}, \frac{b^{\prime}-b}{1-b}\right],\left[\frac{c^{\prime}}{c}, \frac{d^{\prime}}{d}\right]\right)} \\
& =\lim _{\substack{a^{\prime} \rightarrow a \\
b^{\prime} \rightarrow b}}\left(\left[\frac{f_{1}\left(I^{\prime}\right)-f_{1}(I)}{1-f_{1}(I)} \frac{1-a}{a^{\prime}-a}, \frac{f_{2}\left(I^{\prime}\right)-f_{2}(I)}{1-f_{2}(I)} \frac{1-b}{b^{\prime}-b}\right],\right. \\
& \begin{array}{l}
c^{\prime} \rightarrow c \\
d^{\prime} \rightarrow d
\end{array} \\
& \left.\left[\frac{\frac{g_{1}\left(I^{\prime}\right)}{g_{1}(I)}-\frac{c^{\prime}}{c}}{1-\frac{c^{\prime}}{c}}, \frac{\frac{g_{2}\left(I^{\prime}\right)}{g_{2}(I)}-\frac{d^{\prime}}{d}}{1-\frac{d^{\prime}}{d}}\right]\right) \\
& =\left(\left[\lim _{\substack{a^{\prime} \rightarrow a \\
b^{\prime} \rightarrow b \\
c^{\prime} \rightarrow c \\
d^{\prime} \rightarrow d}} \frac{f_{1}\left(I^{\prime}\right)-f_{1}(I)}{1-f_{1}(I)} \frac{1-a}{a^{\prime}-a}, \lim _{\begin{array}{l}
a^{\prime} \rightarrow a \\
b^{\prime} \rightarrow b \\
c^{\prime} \rightarrow c \\
d^{\prime} \rightarrow d
\end{array}} \frac{f_{2}\left(I^{\prime}\right)-f_{2}(I)}{1-f_{2}(I)} \frac{1-b}{b^{\prime}-b}\right),\right. \\
& \left.\left[\lim _{\substack{a^{\prime} \rightarrow a \\
b^{\prime} \rightarrow b \\
c^{\prime} \rightarrow c \\
d^{\prime} \rightarrow d}} \frac{\frac{g_{1}\left(I^{\prime}\right)}{g_{1}(I)}-\frac{c^{\prime}}{c}}{1-\frac{c^{\prime}}{c}}, \lim _{\substack{a^{\prime} \rightarrow a \\
b^{\prime} \rightarrow b \\
c^{\prime} \rightarrow c \\
d^{\prime} \rightarrow d}} \frac{\frac{g_{2}\left(I^{\prime}\right)}{g_{2}(I)}-\frac{d^{\prime}}{d}}{1-\frac{d^{\prime}}{d}}\right]\right)
\end{aligned}
$$

We first consider the left endpoint of the membership degree interval:

$$
\begin{gathered}
\lim _{\substack{a^{\prime} \rightarrow a \\
b \rightarrow a \\
c^{\prime} \rightarrow c \\
d^{\prime} \rightarrow d}} \frac{f_{1}\left(I^{\prime}\right)-f_{1}(I)}{1-f_{1}(I)} \frac{1-a}{a^{\prime}-a}=\lim _{\substack{a^{\prime} \rightarrow a \\
b^{\prime} \rightarrow b \\
d^{\prime} \rightarrow c}} \frac{1-a}{1-f_{1}(I)} \frac{f_{1}\left(I^{\prime}\right)-f_{1}(I)}{a^{\prime}-a} \\
=\lim _{\substack{d^{\prime} \rightarrow a \\
b^{\prime} \rightarrow b \\
c^{\prime} \rightarrow c}} \frac{1-a}{1-f_{1}(I)}\left[\frac{f_{1}\left(a^{\prime}, b^{\prime}, c^{\prime}, d^{\prime}\right)}{a^{\prime}-a}-\frac{f_{1}\left(a, b^{\prime}, c^{\prime}, d^{\prime}\right)}{a^{\prime}-a}+\right. \\
\left(\frac{f_{1}\left(a, b^{\prime}, c^{\prime}, d^{\prime}\right)}{b^{\prime}-b}-\frac{f_{1}\left(a, b, c^{\prime}, d^{\prime}\right)}{b^{\prime}-b}\right) \frac{b^{\prime}-b}{a^{\prime}-a}+ \\
\left(\frac{f_{1}\left(a, b, c^{\prime}, d^{\prime}\right)}{c^{\prime}-c}-\frac{f_{1}\left(a, b, c, d^{\prime}\right)}{c^{\prime}-c}\right) \frac{c^{\prime}-c}{a^{\prime}-a}+ \\
\left.\left(\frac{f_{1}\left(a, b, c, d^{\prime}\right)}{d^{\prime}-d}-\frac{f_{1}(a, b, c, d)}{d^{\prime}-d}\right) \frac{d^{\prime}-d}{a^{\prime}-a}\right]
\end{gathered}
$$




$$
\begin{aligned}
& =\frac{1-a}{1-f_{1}(I)}\left(\frac{\partial f_{1}(I)}{\partial a}+\frac{\partial f_{1}(I)}{\partial b} \lim _{\begin{array}{l}
a^{\prime} \rightarrow a \\
b^{\prime} \rightarrow b \\
c^{\prime} \rightarrow c \\
d^{\prime} \rightarrow d
\end{array}} \frac{b^{\prime}-b}{a^{\prime}-a}+\right. \\
& \left.\frac{\partial f_{1}(I)}{\partial c} \lim _{\substack{a^{\prime} \rightarrow a \\
b^{\prime} \rightarrow b \\
c^{\prime} \rightarrow c \\
d^{\prime} \rightarrow d}} \frac{c^{\prime}-c}{a^{\prime}-a}+\frac{\partial f_{1}(I)}{\partial d} \lim _{\begin{array}{l}
a^{\prime} \rightarrow a \\
b^{\prime} \rightarrow b \\
c^{\prime} \rightarrow c \\
d^{\prime} \rightarrow d
\end{array}} \frac{d^{\prime}-d}{a^{\prime}-a}\right)
\end{aligned}
$$

Similarly, we can get the right endpoint of the membership degree interval:

$$
\begin{aligned}
& \lim _{\substack{a^{\prime} \rightarrow a \\
b^{\prime} \rightarrow b}} \frac{f_{2}\left(I^{\prime}\right)-f_{2}(I)}{1-f_{2}(I)} \frac{1-b}{b^{\prime}-b}=\lim _{\substack{a^{\prime} \rightarrow a \\
b^{\prime} \rightarrow b \\
c^{\prime} \rightarrow \rightarrow}} \frac{1-b}{1-f_{2}(I)} \frac{f_{2}\left(I^{\prime}\right)-f_{2}(I)}{b^{\prime}-b} \\
& \begin{array}{ll}
c^{\prime} \rightarrow c & c^{\prime} \rightarrow c \\
d^{\prime} \rightarrow d & d^{\prime} \rightarrow d
\end{array} \\
& =\frac{1-b}{1-f_{2}(I)}\left(\frac{\partial f_{2}(I)}{\partial b}+\frac{\partial f_{2}(I)}{\partial a} \lim _{\begin{array}{l}
a^{\prime} \rightarrow a \\
b^{\prime} \rightarrow b \\
c^{\prime} \rightarrow c \\
d^{\prime} \rightarrow d
\end{array}} \frac{a^{\prime}-a}{b^{\prime}-b}+\right. \\
& \left.\frac{\partial f_{2}(I)}{\partial c} \lim _{\substack{a^{\prime} \rightarrow a \\
b^{\prime} \rightarrow b \\
c^{\prime} \rightarrow c \\
d^{\prime} \rightarrow d}} \frac{c^{\prime}-c}{b^{\prime}-b}+\frac{\partial f_{2}(I)}{\partial d} \lim _{\substack{a^{\prime} \rightarrow a \\
b^{\prime} \rightarrow b \\
c^{\prime} \rightarrow c \\
d^{\prime} \rightarrow d}} \frac{d^{\prime}-d}{b^{\prime}-b}\right)
\end{aligned}
$$

Next we calculate the part of the non-membership interval. We first consider the left endpoint of the nonmembership degree interval:

$$
\begin{aligned}
& \lim _{\substack{a^{\prime} \rightarrow a \\
b^{\prime} \rightarrow b \\
c^{\prime} \rightarrow c \\
d^{\prime} \rightarrow d}} \frac{\frac{g_{1}\left(I^{\prime}\right)}{g_{1}(I)}-\frac{c^{\prime}}{c}}{1-\frac{c^{\prime}}{c}}=\lim _{\substack{a^{\prime} \rightarrow a \\
b^{\prime} \rightarrow b \\
c^{\prime} \rightarrow c \\
d^{\prime} \rightarrow d}}\left[\frac{c}{g_{1}(I)} \times \frac{g_{1}\left(I^{\prime}\right)}{c-c^{\prime}}-\frac{c^{\prime}}{c-c^{\prime}}\right] \\
& =\lim _{\substack{a^{\prime} \rightarrow a \\
b^{\prime} \rightarrow b \\
c^{\prime} \rightarrow c}}\left\{\frac { c } { d ^ { \prime } \rightarrow d } \left[\frac{g_{1}\left(a^{\prime}, b^{\prime}, c^{\prime}, d^{\prime}\right)-g_{1}\left(a^{\prime}, b^{\prime}, c, d^{\prime}\right)}{c-c^{\prime}}\right.\right. \\
& +\frac{g_{1}\left(a^{\prime}, b^{\prime}, c, d^{\prime}\right)-g_{1}\left(a, b^{\prime}, c, d^{\prime}\right)}{a-a^{\prime}} \frac{a-a^{\prime}}{c-c^{\prime}} \\
& +\frac{g_{1}\left(a, b^{\prime}, c, d^{\prime}\right)-g_{1}\left(a, b, c, d^{\prime}\right)}{b-b^{\prime}} \frac{b-b^{\prime}}{c-c^{\prime}} \\
& \left.\left.+\frac{g_{1}\left(a, b, c, d^{\prime}\right)-g_{1}(a, b, c, d)}{d-d^{\prime}} \frac{d-d^{\prime}}{c-c^{\prime}}+\frac{g_{1}(a, b, c, d)}{c-c^{\prime}}\right]-\frac{c^{\prime}}{c-c^{\prime}}\right\}
\end{aligned}
$$

$$
\begin{aligned}
& =1-\frac{c}{g_{1}(I)}\left[\frac{\partial g_{1}(I)}{\partial c}+\frac{\partial g_{1}(I)}{\partial a} \lim _{\begin{array}{l}
a^{\prime} \rightarrow a \\
b^{\prime} \rightarrow b \\
c^{\prime} \rightarrow c \\
d^{\prime} \rightarrow d
\end{array}} \frac{a^{\prime}-a}{c^{\prime}-c}+\right. \\
& \left.\frac{\partial g_{1}(I)}{\partial b} \lim _{\substack{a^{\prime} \rightarrow a \\
b^{\prime} \rightarrow b \\
c^{\prime} \rightarrow c \\
d^{\prime} \rightarrow d}} \frac{b^{\prime}-b}{c^{\prime}-c}+\frac{\partial g_{1}(I)}{\partial d} \lim _{\begin{array}{l}
a^{\prime} \rightarrow a \\
b^{\prime} \rightarrow b \\
c^{\prime} \rightarrow c \\
d^{\prime} \rightarrow d
\end{array}} \frac{d^{\prime}-d}{c^{\prime}-c}\right]
\end{aligned}
$$

Likewise, we get the right endpoint of the nonmembership degree interval as follows:

$$
\begin{aligned}
& \lim _{\substack{a^{\prime} \rightarrow a \\
b^{\prime} \rightarrow b \\
c^{\prime} \rightarrow c \\
d^{\prime} \rightarrow d}} \frac{\frac{g_{2}\left(I^{\prime}\right)}{g_{2}(I)}-\frac{d^{\prime}}{d}}{1-\frac{d^{\prime}}{d}}=\lim _{\substack{a^{\prime} \rightarrow a \\
b^{\prime} \rightarrow b \\
c^{\prime} \rightarrow c \\
d^{\prime} \rightarrow d}}\left[\frac{d}{g_{2}(I)} \times \frac{g_{2}\left(I^{\prime}\right)}{d-d^{\prime}}-\frac{d^{\prime}}{d-d^{\prime}}\right] \\
& =1-\frac{d}{g_{2}(I)}\left[\frac{\partial g_{2}(I)}{\partial d}+\frac{\partial g_{2}(I)}{\partial a} \lim _{\begin{array}{l}
a^{\prime} \rightarrow a \\
b^{\prime} \rightarrow b \\
c^{\prime} \rightarrow c \\
d^{\prime} \rightarrow d
\end{array}} \frac{a^{\prime}-a}{d^{\prime}-d}+\right. \\
& \left.\frac{\partial g_{2}(I)}{\partial b} \lim _{\substack{a^{\prime} \rightarrow a \\
b^{\prime} \rightarrow b \\
c^{\prime} \rightarrow c \\
d^{\prime} \rightarrow d}} \frac{b^{\prime}-b}{d^{\prime}-d}+\frac{\partial g_{2}(I)}{\partial c} \lim _{\begin{array}{l}
a^{\prime} \rightarrow a \\
b^{\prime} \rightarrow b \\
c^{\prime} \rightarrow c \\
d^{\prime} \rightarrow d
\end{array}} \frac{c^{\prime}-c}{d^{\prime}-d}\right]
\end{aligned}
$$

To ensure the derivative to be only the IVIFV, which doesn't change with $\tilde{\alpha}$, we have

$$
\left\{\begin{array}{l}
\frac{\partial f_{1}(a, b, c, d)}{\partial b}=\frac{\partial f_{1}(a, b, c, d)}{\partial c}=\frac{\partial f_{1}(a, b, c, d)}{\partial d}=0 \\
\frac{\partial f_{2}(a, b, c, d)}{\partial a}=\frac{\partial f_{2}(a, b, c, d)}{\partial c}=\frac{\partial f_{2}(a, b, c, d)}{\partial d}=0 \\
\frac{\partial g_{1}(a, b, c, d)}{\partial a}=\frac{\partial g_{1}(a, b, c, d)}{\partial b}=\frac{\partial g_{1}(a, b, c, d)}{\partial d}=0 \\
\frac{\partial g_{2}(a, b, c, d)}{\partial a}=\frac{\partial g_{2}(a, b, c, d)}{\partial b}=\frac{\partial g_{2}(a, b, c, d)}{\partial c}=0
\end{array}\right.
$$

and 


$$
\left\{\begin{aligned}
0 & \leq \frac{1-a}{1-f_{1}(a, b, c, d)} \frac{\partial f_{1}(a, b, c, d)}{\partial a} \leq 1 \\
0 & \leq \frac{c}{g_{1}(a, b, c, d)} \frac{\partial g_{1}(a, b, c, d)}{\partial c} \leq 1 \\
0 \leq \frac{1-b}{1-f_{2}(a, b, c, d)} \frac{\partial f_{2}(a, b, c, d)}{\partial b} & \leq \frac{d}{g_{2}(a, b, c, d)} \frac{\partial g_{2}(a, b, c, d)}{\partial d} \leq 1
\end{aligned}\right.
$$

Thus $F(\tilde{\alpha})=\left(\left[f_{1}(a), f_{2}(b)\right],\left[g_{1}(c), g_{2}(d)\right]\right)$, and then

$$
\begin{aligned}
\frac{d F(\tilde{\alpha})}{d \tilde{\alpha}}= & \left(\left[\frac{1-a}{1-f_{1}(a)} f_{1}^{\prime}(a), \frac{1-b}{1-f_{2}(b)} f_{2}^{\prime}(b)\right],\right. \\
& {\left.\left[1-\frac{c}{g_{1}(c)} g_{1}^{\prime}(c), 1-\frac{d}{g_{2}(d)} g_{2}^{\prime}(d)\right]\right) }
\end{aligned}
$$

which completes the proof.

In a similar way, we can get the derivative of $F(\tilde{\alpha})$ at $\tilde{\alpha}$ in the subtraction direction. When $\tilde{\alpha}^{\prime} \in \tilde{S}^{\ominus}(\tilde{\alpha}, F)$, we have

$$
\begin{aligned}
& \frac{d F(\tilde{\alpha})}{d \tilde{\alpha}}=\lim _{\tilde{\alpha}^{\prime} \rightarrow \tilde{\alpha}^{\ominus}} \frac{F(\tilde{\alpha}) \ominus F\left(\tilde{\alpha}^{\prime}\right)}{\tilde{\alpha} \ominus \tilde{\alpha}^{\prime}} \\
& =\left(\left[\frac{1-a}{1-f_{1}(a)} f_{1}^{\prime}(a), \frac{1-b}{1-f_{2}(b)} f_{2}^{\prime}(b)\right],\left[1-\frac{c}{g_{1}(c)} g_{1}^{\prime}(c), 1-\frac{d}{g_{2}(d)} g_{2}^{\prime}(d)\right]\right)
\end{aligned}
$$

If the value $\frac{d F(\tilde{\alpha})}{d \tilde{\alpha}}$ is an IVIFV, then we call it the derivative of $F(\tilde{\alpha})$ at $\tilde{\alpha}$ in the subtraction direction.

We find that the derivative values of $F(\tilde{\alpha})$ at $\tilde{\alpha}$ in the addition and subtraction directions are exactly the same if $f_{1}, f_{2}$ and $g_{1}, g_{2}$ are derivable. So we shall unify the two kinds of derivatives into one.

Definition 26. If the IVIFF $F(\tilde{\alpha})$ is differentiable at $\tilde{\alpha}$ in its addition and subtraction directions, then we call

$$
\begin{aligned}
\frac{d F(\tilde{\alpha})}{d \tilde{\alpha}}= & \left(\left[\frac{1-a}{1-f_{1}(a)} f_{1}^{\prime}(a), \frac{1-b}{1-f_{2}(b)} f_{2}^{\prime}(b)\right],\right. \\
& {\left.\left[1-\frac{c}{g_{1}(c)} g_{1}^{\prime}(c), 1-\frac{d}{g_{2}(d)} g_{2}^{\prime}(d)\right]\right) }
\end{aligned}
$$

the subtraction derivative of $F(\tilde{\alpha})$ at $\tilde{\alpha}$.

Theorem 2 gives a condition for the existence of an IVIFF's subtraction derivative. This is just like the " $\mathrm{C}-\mathrm{R}$ condition" in the complex number field.

In what follows, we shall study the subtraction derivatives of some special IVIFFs:
(1) If $F(\tilde{X})=\tilde{\alpha}_{0}=\left(\left[a_{0}, b_{0}\right],\left[c_{0}, d_{0}\right]\right)$, then

$$
\begin{aligned}
& f_{1}(a)=a_{0}, f_{2}(b)=b_{0}, g_{1}(c)=c_{0}, \\
& g_{2}(d)=d_{0}, \frac{d F(\tilde{X})}{d \tilde{X}}=([0,0],[1,1])
\end{aligned}
$$

(2)If

$F(\tilde{X})=\tilde{X} \oplus \tilde{\alpha}_{0}=\left(\left[a+a_{0}-a a_{0}, b+b_{0}-b b_{0}\right],\left[c c_{0}, d d_{0}\right]\right)$ , then

$$
\begin{aligned}
& f_{1}(a)=a+a_{0}-a a_{0}, f_{2}(b)=b+b_{0}-b b_{0}, g_{1}(c)=c c_{0}, g_{2}(d)=d d_{0} \\
& \frac{d F(\tilde{X})}{d \tilde{X}}=\left(\left[\frac{1-a}{1-a-a_{0}+a a_{0}}\left(1-a_{0}\right), \frac{1-b}{1-b-b_{0}+b b_{0}}\left(1-b_{0}\right)\right],\right.
\end{aligned}
$$$$
\left.\left[1-\frac{c}{c c_{0}} c_{0}, 1-\frac{d}{d d_{0}} d_{0}\right]\right)=([1,1],[0,0])
$$

(3)If

$$
F(\tilde{X})=\tilde{\alpha}_{0} \otimes \tilde{X}=\left(\left[a_{0} a, b_{0} b\right],\left[c_{0}+c-c_{0} c, d_{0}+d-d_{0} d\right]\right)
$$

, then

$$
\begin{gathered}
f_{1}(a)=a_{0} a, f_{2}(b)=b_{0} b \\
g_{1}(c)=c_{0}+c-c_{0} c, g_{2}(d)=d_{0}+d-d_{0} d
\end{gathered}
$$

$\frac{d F(\tilde{X})}{d \tilde{X}}=\left(\left[\frac{1-a}{1-a_{0} a} a_{0}, \frac{1-b}{1-b_{0} b} b_{0}\right]\right.$,

$\left.\left[1-\frac{c}{c_{0}+c-c_{0} c}\left(1-c_{0}\right), 1-\frac{d}{d_{0}+d-d_{0} d}\left(1-d_{0}\right)\right]\right)$
$=\left(\left[\frac{a_{0}-a_{0} a}{1-a_{0} a}, \frac{b_{0}-b_{0} b}{1-b_{0} b}\right],\left[\frac{c_{0}}{c_{0}+c-c_{0} c}, \frac{d_{0}}{d_{0}+d-d_{0} d}\right]\right)$
$=\left(\left[a_{0}, b_{0}\right],\left[c_{0}, d_{0}\right]\right) \otimes$
$\left(\left[\frac{1-a}{1-a_{0} a}, \frac{1-b}{1-b_{0} b}\right],\left[1-\frac{c}{c_{0}+c-c_{0} c}, 1-\frac{d}{d_{0}+d-d_{0} d}\right]\right)$

(a) When $\tilde{\alpha}_{0}=\left(\left[a_{0}, b_{0}\right],\left[c_{0}, d_{0}\right]\right)=([1,1],[0,0])$, we get

$$
\frac{d F(\tilde{X})}{d \tilde{X}}=\left(\left[\frac{1-a}{1-a}, \frac{1-b}{1-b}\right],[0,0]\right)=([1,1],[0,0])
$$

(b) When $\tilde{\alpha}_{0}=\left(\left[a_{0}, b_{0}\right],\left[c_{0}, d_{0}\right]\right)=([0,0],[1,1])$, we have 


$$
\begin{aligned}
\frac{d F(\tilde{X})}{d \tilde{X}} & =\left([0,0],\left[\frac{1}{c_{0}+c-c_{0} c}, \frac{1}{d_{0}+d-d_{0} d}\right]\right) \\
& =([0,0],[1,1])
\end{aligned}
$$

\section{Because}

$\frac{a_{0}-a_{0} a}{1-a_{0} a}=a_{0} \frac{1-a}{1-a_{0} a} \leq a_{0}, \frac{b_{0}-b_{0} b}{1-b_{0} b}=b_{0} \frac{1-b}{1-b_{0} b} \leq b_{0}$

$$
\text { and } \begin{aligned}
& \frac{c_{0}}{c+c_{0}-c_{0} c}=c_{0} \frac{1}{c+c_{0}(1-c)} \geq c_{0}, \\
& \frac{d_{0}}{d+d_{0}-d_{0} d}=d_{0} \frac{1}{d+d_{0}(1-d)} \geq d_{0}
\end{aligned}
$$

Then we can get

$\left(\left[\frac{a_{0}-a_{0} a}{1-a_{0} a}, \frac{b_{0}-b_{0} b}{1-b_{0} b}\right],\left[\frac{c_{0}}{c_{0}+c-c_{0} c}, \frac{d_{0}}{d_{0}+d-d_{0} d}\right]\right)$

$\leq_{L}\left(\left[a_{0}, b_{0}\right],\left[c_{0}, d_{0}\right]\right)$

(4) If

$F(\tilde{X})=\lambda \cdot \tilde{X}=\left(\left[1-(1-a)^{\lambda}, 1-(1-b)^{\lambda}\right],\left[c^{\lambda}, d^{\lambda}\right]\right), 0<\lambda \leq 1$ , then

$$
\begin{aligned}
& f_{1}(a)=1-(1-a)^{\lambda}, f_{2}(b)=1-(1-b)^{\lambda}, g_{1}(c)=c^{\lambda}, g_{2}(d)=d^{\lambda} \\
& \begin{aligned}
\frac{d F(\tilde{X})}{d \tilde{X}} & =\left(\left[\frac{1-a}{(1-a)^{\lambda}} \lambda(1-a)^{\lambda-1}, \frac{1-b}{(1-b)^{\lambda}} \lambda(1-b)^{\lambda-1}\right],[1-\lambda, 1-\lambda]\right) \\
& =([\lambda, \lambda],[1-\lambda, 1-\lambda])
\end{aligned}
\end{aligned}
$$

The examples (5) and (6) below show that in interval-valued intuitionistic fuzzy environment, for any two functions $F(\tilde{X})=K \tilde{X}+\tilde{\alpha} \quad$ and $G(\tilde{X})=K \tilde{X}+\tilde{\beta}$ whether $K$ is an IVIFV or a positive real number, we have $\frac{d F(\tilde{X})}{d \tilde{X}}=\frac{d G(\tilde{X})}{d \tilde{X}}$. This conclusion is similar to the one in the real number field.

(5) If $G(\tilde{X})=\tilde{\alpha} \otimes \tilde{X} \oplus \tilde{\beta}$, then

$\tilde{\alpha} \otimes \tilde{X} \oplus \tilde{\beta}=\left(\left[a_{\alpha} a+a_{\beta}-a_{\alpha} a_{\beta} a, b_{\alpha} b+b_{\beta}-b_{\alpha} b_{\beta} b\right]\right.$,

$$
\left.\left[c_{\beta} c+c_{\alpha} c_{\beta}-c_{\alpha} c_{\beta} c, d_{\beta} d+d_{\alpha} d_{\beta}-d_{\alpha} d_{\beta} d\right]\right)
$$

$$
f_{1}(a)=a_{\alpha} a+a_{\beta}-a_{\alpha} a_{\beta} a, f_{2}(b)=b_{\alpha} b+b_{\beta}-b_{\alpha} b_{\beta} b
$$

$g_{1}(c)=c_{\beta} c+c_{\alpha} c_{\beta}-c_{\alpha} c_{\beta} c, g_{2}(d)=d_{\beta} d+d_{\alpha} d_{\beta}-d_{\alpha} d_{\beta} d$

$$
\begin{aligned}
\frac{d G(X)}{d X}= & \left(\left[\frac{1-a}{1-\left(a_{\alpha} a+a_{\beta}-a_{\alpha} a_{\beta} a\right)}\left(a_{\alpha}-a_{\alpha} a_{\beta}\right),\right.\right. \\
& \left.\frac{1-b}{1-\left(b_{\alpha} b+b_{\beta}-b_{\alpha} b_{\beta} b\right)}\left(b_{\alpha}-b_{\alpha} b_{\beta}\right)\right], \\
& {\left[1-\frac{c}{c_{\beta} c+c_{\alpha} c_{\beta}-c_{\alpha} c_{\beta} c}\left(c_{\beta}-c_{\alpha} c_{\beta}\right),\right.} \\
& \left.\left.1-\frac{d}{d_{\beta} d+d_{\alpha} d_{\beta}-d_{\alpha} d_{\beta} d}\left(d_{\beta}-d_{\alpha} d_{\beta}\right)\right]\right) \\
= & \left(\left[\frac{a_{\alpha}-a_{\alpha} a}{1-a_{\alpha} a}, \frac{b_{\alpha}-b_{\alpha} b}{1-b_{\alpha} b}\right],\left[\frac{c_{\alpha}}{c_{\alpha}+c-c_{\alpha} c}, \frac{d_{\alpha}}{d_{\alpha}+d-d_{\alpha} d}\right]\right)
\end{aligned}
$$

Let $F(\tilde{X})=\tilde{\alpha} \otimes \tilde{X}$, then we get $\frac{d G(\tilde{X})}{d \tilde{X}}=\frac{d F(\tilde{X})}{d \tilde{X}}$.

(6) If $G(\tilde{X})=\lambda \tilde{X} \oplus \tilde{\beta}, \lambda>0$, then

$$
\begin{gathered}
\lambda \tilde{X} \oplus \tilde{\beta}=\left(\left[1-(1-a)^{\lambda}+a_{\beta}(1-a)^{\lambda},\right.\right. \\
\left.\left.1-(1-b)^{\lambda}+b_{\beta}(1-b)^{\lambda}\right],\left[c^{\lambda} c_{\beta}, d^{\lambda} d_{\beta}\right]\right) \\
f_{1}(a)=1-(1-a)^{\lambda}+a_{\beta}(1-a)^{\lambda}, \quad f_{2}(b)=1-(1-b)^{\lambda}+b_{\beta}(1-b)^{\lambda} \\
g_{1}(c)=c^{\lambda} c_{\beta}, \quad g_{2}(d)=d^{\lambda} d_{\beta} \\
\frac{d G(\tilde{X})}{d \tilde{X}}=([\lambda, \lambda],[1-\lambda, 1-\lambda])
\end{gathered}
$$

Let $F(\tilde{X})=\lambda \tilde{X}$, then we get $\frac{d G(\tilde{X})}{d \tilde{X}}=\frac{d F(\tilde{X})}{d \tilde{X}}$.

At last, as a special IVIFF, we examine the derivative of the IIFWA operator:

(7) If

$$
\begin{aligned}
& \text { IIFWA }_{\omega}\left(\tilde{\alpha}_{1}, \tilde{\alpha}_{2}, \ldots, \tilde{\alpha}_{n}\right)= \\
& \left(\left[1-\prod_{j=1}^{n}\left(1-a_{j}\right)^{\omega_{j}}, 1-\prod_{j=1}^{n}\left(1-b_{j}\right)^{\omega_{j}}\right],\left[\prod_{j=1}^{n} c_{j}^{\omega_{j}}, \prod_{j=1}^{n} d_{j}^{\omega_{j}}\right]\right)
\end{aligned}
$$

then

$$
\begin{aligned}
& f_{1}\left(a_{j}\right)=1-\prod_{j=1}^{n}\left(1-a_{j}\right)^{\omega_{j}}, f_{2}\left(b_{j}\right)=1-\prod_{j=1}^{n}\left(1-b_{j}\right)^{\omega_{j}} ; \\
& g_{1}\left(c_{j}\right)=\prod_{j=1}^{n} c_{j}^{\omega_{j}}, g_{2}\left(d_{j}\right)=\prod_{j=1}^{n} d_{j}^{\omega_{j}}
\end{aligned}
$$

where $\tilde{\alpha}_{j}=\left(\left[a_{j}, b_{j}\right],\left[c_{j}, d_{j}\right]\right)$, and then

$$
\frac{d\left(\operatorname{IIFWA} A_{\omega}\left(\tilde{\alpha}_{1}, \tilde{\alpha}_{2}, \cdots, \tilde{\alpha}_{n}\right)\right)}{d \tilde{\alpha}_{i}}
$$




$$
\begin{aligned}
& =\left(\left[\frac{1-a_{j}}{\prod_{j=1}^{n}\left(1-a_{j}\right)^{\omega_{j}}} \omega_{\substack { i \\
\begin{subarray}{c}{j=1 \\
j \neq i{ i \\
\begin{subarray} { c } { j = 1 \\
j \neq i } }\end{subarray}}^{n}\left(1-a_{j}\right)^{\omega_{j}}\left(1-a_{j}\right)^{\omega_{i}-1},\right.\right. \\
& \left.\frac{1-b_{j}}{\prod_{j=1}^{n}\left(1-b_{j}\right)^{\omega_{j}}} \omega_{i} \prod_{\substack{j=1 \\
j \neq i}}^{n}\left(1-b_{j}\right)^{\omega_{j}}\left(1-b_{j}\right)^{\omega_{i}-1}\right], \\
& \left.\left[1-\frac{c_{j}}{\prod_{j=1}^{n} c_{j}^{\omega_{j}}} \omega_{i} \prod_{\substack{j=1 \\
j \neq i}}^{n} c_{j}^{\omega_{j}} \cdot c_{j}^{\omega_{i}-1}, 1-\frac{d_{j}}{\prod_{j=1}^{n} d_{j}^{\omega_{j}}} \omega_{i} \prod_{\substack{j=1 \\
j \neq i}}^{n} d_{j}^{\omega_{j}} \cdot d_{j}^{\omega_{i}-1}\right]\right) \\
& =\left(\left[\omega_{i}, \omega_{i}\right],\left[1-\omega_{i}, 1-\omega_{i}\right]\right)
\end{aligned}
$$

As defined before, the change value for an IVIFV has four different change directions: the addition, subtraction, multiplication and division directions. We have discussed the addition and subtraction derivatives for an IVIFF. In the following, we shall consider the differentiability of an IVIFF in the multiplication and division directions:

Definition 27. If $\lim _{\tilde{\alpha}^{\prime} \rightarrow \tilde{\alpha}^{\odot}}\left(\frac{F(\tilde{\alpha})}{F(\tilde{\alpha})} \ominus \frac{\tilde{\alpha}}{\tilde{\alpha}^{\prime}}\right)$ is an IVIFV, then we call $F(\tilde{\alpha})$ to be differentiable at $\tilde{\alpha}$ in the division direction, and the limit value is called the derivative of $F(\tilde{\alpha})$ at $\tilde{\alpha}$.

$$
\begin{aligned}
& \text { Theorem 3. Suppose that } \\
& F(\tilde{\alpha})=\left(\left[f_{1}(a, b, c, d), f_{2}(a, b, c, d)\right],\left[g_{1}(a, b, c, d), g_{2}(a, b, c, d)\right]\right) \\
& \text { is an IVIFF of } \tilde{\alpha}=([a, b],[c, d]) \text {, then } F(\tilde{\alpha}) \text { is }
\end{aligned}
$$

differentiable at $\tilde{\alpha}$ in the division direction if and only if

$$
\left\{\begin{array}{l}
\frac{\partial f_{1}(a, b, c, d)}{\partial b}=\frac{\partial f_{1}(a, b, c, d)}{\partial c}=\frac{\partial f_{1}(a, b, c, d)}{\partial d}=0 \\
\frac{\partial f_{2}(a, b, c, d)}{\partial a}=\frac{\partial f_{2}(a, b, c, d)}{\partial c}=\frac{\partial f_{2}(a, b, c, d)}{\partial d}=0 \\
\frac{\partial g_{1}(a, b, c, d)}{\partial a}=\frac{\partial g_{1}(a, b, c, d)}{\partial b}=\frac{\partial g_{1}(a, b, c, d)}{\partial d}=0 \\
\frac{\partial g_{2}(a, b, c, d)}{\partial a}=\frac{\partial g_{2}(a, b, c, d)}{\partial b}=\frac{\partial g_{2}(a, b, c, d)}{\partial c}=0
\end{array}\right.
$$

and

$$
\left\{\begin{array}{c}
0 \leq \frac{a}{f_{1}(a, b, c, d)} \frac{\partial f_{1}(a, b, c, d)}{\partial a} \leq 1 \\
0 \leq \frac{1-c}{1-g_{1}(a, b, c, d)} \frac{\partial g_{1}(a, b, c, d)}{\partial c} \leq 1 \\
0 \leq \frac{1-d}{1-g_{2}(a, b, c, d)} \frac{\partial g_{2}(a, b, c, d)}{\partial d} \leq \frac{b}{f_{2}(a, b, c, d)} \frac{\partial f_{2}(a, b, c, d)}{\partial b} \leq 1
\end{array}\right.
$$

Under such sufficient and necessary condition, $F(\tilde{\alpha})$ can be expressed by $F(\tilde{\alpha})=\left(\left[f_{1}(a), f_{2}(b)\right],\left[g_{1}(c), g_{2}(d)\right]\right)$, and then the derivative $F(\tilde{\alpha})$ at $\tilde{\alpha}$ in the division direction can be computed as:

$$
\begin{aligned}
\frac{l F(\tilde{\alpha})}{l \tilde{\alpha}}= & \left(\left[1-\frac{a}{f_{1}(a)} f_{1}^{\prime}(a), 1-\frac{b}{f_{2}(b)} f_{2}^{\prime}(b)\right],\right. \\
& \left.\left.\frac{1-c}{1-g_{1}(c)} g_{1}^{\prime}(c), \frac{1-d}{1-g_{2}(d)} g_{2}^{\prime}(d)\right]\right)
\end{aligned}
$$

When $\alpha^{\prime} \in \tilde{S}^{\otimes}(\alpha, F)$, similarly, we have

$$
\begin{aligned}
\frac{l F(\tilde{\alpha})}{l \tilde{\alpha}}= & \lim _{\tilde{\alpha}^{\prime} \rightarrow \tilde{\alpha}^{\otimes}}\left(\frac{F\left(\tilde{\alpha}^{\prime}\right)}{F(\tilde{\alpha})} \Theta \frac{\tilde{\alpha}^{\prime}}{\tilde{\alpha}}\right) \\
= & \left(\left[1-\frac{a}{f_{1}(a)} f_{1}^{\prime}(a), 1-\frac{b}{f_{2}(b)} f_{2}^{\prime}(b)\right],\right. \\
& {\left.\left[\frac{1-c}{1-g_{1}(c)} g_{1}^{\prime}(c), \frac{1-d}{1-g_{2}(d)} g_{2}^{\prime}(d)\right]\right) }
\end{aligned}
$$

If the limit value is an IVIFV, then we call it the derivative of $F(\tilde{\alpha})$ at $\tilde{\alpha}$ in the multiplication direction.

As we can see, there are exactly the same derivative values in the division and multiplication directions if the functions $f_{1}, f_{2}$ and $g_{1}, g_{2}$ are derivable. So we also unify the two derivatives into one:

Definition 28. If the IVIFF $F(\tilde{\alpha})$ is differentiable at $\tilde{\alpha}$ in the division and multiplication directions, then

$$
\begin{aligned}
\frac{l F(\tilde{\alpha})}{l \tilde{\alpha}}=( & {\left[1-\frac{a}{f_{1}(a)} f_{1}^{\prime}(a), 1-\frac{b}{f_{2}(b)} f_{2}^{\prime}(b)\right], } \\
& {\left.\left[\frac{1-c}{1-g_{1}(c)} g_{1}^{\prime}(c), \frac{1-d}{1-g_{2}(d)} g_{2}^{\prime}(d)\right]\right) }
\end{aligned}
$$

is called the division derivative of $F(\tilde{\alpha})$ at $\tilde{\alpha}$.

In the following, let's see the division derivatives of some special IVIFFs: 
(1) If $F(\tilde{X})=\tilde{\alpha}_{0}=\left(\left[a_{0}, b_{0}\right],\left[c_{0}, d_{0}\right]\right)$, then

$$
\begin{aligned}
& f_{1}(a)=a_{0}, f_{2}(b)=b_{0}, g_{1}(c)=c_{0}, \\
& g_{2}(d)=d_{0}, \frac{l F(\tilde{X})}{l \tilde{X}}=([1,1],[0,0])
\end{aligned}
$$

(2) If $F(\tilde{X})=\tilde{\alpha}_{0} \oplus \tilde{X}$,

$=\left(\left[a_{0}+a-a_{0} a, b_{0}+b-b_{0} b\right],\left[c_{0} c, d_{0} d\right]\right)$ then

$f_{1}(a)=a_{0}+a-a_{0} a, f_{2}(b)=b_{0}+b-b_{0} b$,

$g_{1}(c)=c_{0} c, g_{2}(d)=d_{0} d$

$\frac{l F(\tilde{X})}{l \tilde{X}}=\left(\left[1-\frac{a}{a_{0}+a-a_{0} a}\left(1-a_{0}\right)\right.\right.$,

$$
\left.\left.1-\frac{b}{b_{0}+b-b_{0} b}\left(1-b_{0}\right)\right],\left[\frac{1-c}{1-c_{0} c} c_{0}, \frac{1-d}{1-d_{0} d} d_{0}\right]\right)
$$

$=\left(\left[\frac{a_{0}}{a_{0}+a-a_{0} a}, \frac{b_{0}}{b_{0}+b-b_{0} b}\right],\left[\frac{c_{0}-c_{0} c}{1-c_{0} c}, \frac{d_{0}-d_{0} d}{1-d_{0} d}\right]\right)$

$=\left(\left[a_{0}, b_{0}\right],\left[c_{0}, d_{0}\right]\right)$

$\oplus\left(\left[1-\frac{a}{a_{0}+a-a_{0} a}, 1-\frac{b}{b_{0}+b-b_{0} b}\right],\left[\frac{1-c}{1-c_{0} c}, \frac{1-d}{1-d_{0} d}\right]\right)$

$\geq_{L}\left(\left[a_{0}, b_{0}\right],\left[c_{0}, d_{0}\right]\right)$

(3) If

$F(\tilde{X})=\tilde{\alpha}_{0} \otimes \tilde{X}=\left(\left[a_{0} a, b_{0} b\right],\left[c_{0}+c-c_{0} c, d_{0}+d-d_{0} d\right]\right)$, then

$f_{1}(a)=a_{0} a, f_{2}(b)=b_{0} b$,

$g_{1}(c)=c_{0}+c-c_{0} c, g_{2}(d)=d_{0}+d-d_{0} d$

$\frac{l F(X)}{l X}=\left(\left[1-\frac{a}{a_{0} a} a_{0}, 1-\frac{b}{b_{0} b} b_{0}\right]\right.$,

$\left.\left[\frac{1-c}{1-c-c_{0}+c_{0} c}\left(1-c_{0}\right), \frac{1-d}{1-d-d_{0}+d_{0} d}\left(1-d_{0}\right)\right]\right)$

$=([0,0],[1,1])$

(4) If

$$
\begin{aligned}
F(\tilde{X})=\tilde{X}^{\lambda}= & \left(\left[a^{\lambda}, b^{\lambda}\right],\right. \\
& {\left.\left[1-(1-c)^{\lambda}, 1-(1-d)^{\lambda}\right]\right), 0<\lambda \leq 1 }
\end{aligned}
$$

then

$f_{1}(a)=a^{\lambda}, f_{2}(b)=b^{\lambda}$,

$g_{1}(c)=1-(1-c)^{\lambda}, g_{2}(d)=1-(1-d)^{\lambda}$

$$
\begin{aligned}
& \frac{l F(\tilde{X})}{l \tilde{X}}=([1-\lambda, 1-\lambda], \\
& \left.\left[\frac{1-c}{(1-c)^{\lambda}} \lambda(1-c)^{\lambda-1}, \frac{1-d}{(1-d)^{\lambda}} \lambda(1-d)^{\lambda-1}\right]\right) \\
& =([1-\lambda, 1-\lambda],[\lambda, \lambda])
\end{aligned}
$$

(5) If

$\operatorname{IIFWG}_{\omega}\left(\tilde{\alpha}_{1}, \tilde{\alpha}_{2}, \ldots, \tilde{\alpha}_{n}\right)=$

$$
\left(\left[\prod_{j=1}^{n} a_{j}^{\omega_{j}}, \prod_{j=1}^{n} b_{j}^{\omega_{j}}\right],\left[1-\prod_{j=1}^{n}\left(1-c_{j}\right)^{\omega_{j}}, 1-\prod_{j=1}^{n}\left(1-d_{j}\right)^{\omega_{j}}\right]\right)
$$

then

$$
\begin{aligned}
& f_{1}(a)=\prod_{j=1}^{n} a_{j}^{\omega_{j}}, f_{2}(b)=\prod_{j=1}^{n} b_{j}^{\omega_{j}}, \\
& g_{1}(c)=1-\prod_{j=1}^{n}\left(1-c_{j}\right)^{\omega_{j}}, g_{2}(d)=1-\prod_{j=1}^{n}\left(1-d_{j}\right)^{\omega_{j}} \\
& \frac{l\left(I I F W G_{\omega}\left(\tilde{\alpha}_{1}, \tilde{\alpha}_{2}, \cdots, \tilde{\alpha}_{n}\right)\right)}{l \tilde{\alpha}_{i}}=
\end{aligned}
$$$$
\left(\left[1-\frac{a_{i}}{\prod_{j=1}^{n} a_{j}^{\omega_{j}}} \omega_{i} \prod_{\substack{j=1 \\ j \neq i}}^{n} a_{j}^{\omega_{j}} \cdot a_{i}^{\omega_{i}-1}, 1-\frac{b_{i}}{\prod_{j=1}^{n} b_{j}^{\omega_{j}}} \omega_{i} \prod_{\substack{j=1 \\ j \neq i}}^{n} b_{j}^{\omega_{j}} \cdot b_{i}^{\omega_{i}-1}\right],\right.
$$$$
\left[\frac{1-c_{i}}{\prod_{j=1}^{n}\left(1-c_{j}\right)^{\omega_{j}}} \omega_{i} \prod_{\substack{j=1 \\ j \neq i}}^{n}\left(1-c_{j}\right)^{\omega_{j}}\left(1-c_{i}\right)^{\omega_{i}-1},\right.
$$

$$
\begin{aligned}
& \left.\left.\frac{1-d_{i}}{\prod_{j=1}^{n}\left(1-d_{j}\right)^{\omega_{j}}} \omega_{i} \prod_{\substack{j=1 \\
j \neq i}}^{n}\left(1-d_{j}\right)^{\omega_{j}}\left(1-d_{i}\right)^{\omega_{i}-1}\right]\right) \\
& =\left(\left[1-\omega_{i}, 1-\omega_{i}\right],\left[\omega_{i}, \omega_{i}\right]\right)
\end{aligned}
$$

\section{The differentials of IVIFFs and their applications in approximate calculation}

In real-life applications, we often encounter some complicated functions. If we compute the function value with the function itself, we shall need great effort. In some cases, we only need the approximate function value instead of the precise value. Using the differential, we can do it. In the following, we shall discuss the approximate calculation methods under interval-valued intuitionistic fuzzy environment. 
To begin with, we give the concept of differential for IVIFFs. In the last section, we have defined two kinds of derivatives (the subtraction derivative and the division derivative), so we shall define two differential operations accordingly:

Definition 29. For a given IVIFV $\tilde{\alpha}=([a, b],[c, d])$, we call $A(\tilde{\alpha})=a$ and $B(\tilde{\alpha})=b$ the take-value functions of membership interval and $C_{L}(\tilde{\alpha})=c, D_{R}(\tilde{\alpha})=d$ the take-value functions of nonmembership interval.

In what follows, we give the first kind of differential-the subtraction differential:

\section{Definition}

30.

Assume

that

$\tilde{Y}=F(\tilde{\alpha})=\left(\left[f_{1}(a), f_{2}(b)\right],\left[g_{1}(c), g_{2}(d)\right]\right) \quad$ is $\quad$ an IVIFF and $\Delta \tilde{\alpha}=\tilde{\alpha}^{\prime} \ominus \tilde{\alpha}$, then the concrete form of the subtraction differential of $F(\tilde{\alpha})$ is defined as $\frac{d F(\tilde{\alpha})}{d \tilde{\alpha}} \otimes \Delta \tilde{\alpha}$, denoted by $d \tilde{Y}$, that is,

$$
d \tilde{Y}=\frac{d F(\tilde{\alpha})}{d \tilde{\alpha}} \otimes \Delta \tilde{\alpha}
$$

The subtraction differential of the independent variable $\tilde{\alpha}$ is equal to $\Delta \tilde{\alpha}$. In fact,

$d \tilde{\alpha}=d F(\tilde{\alpha})=\frac{d F(\tilde{\alpha})}{d \tilde{\alpha}} \otimes \Delta \tilde{\alpha}=([1,1],[0,0]) \otimes \Delta \tilde{\alpha}=\Delta \tilde{\alpha}$

So the subtraction differential of arbitrary IVIFF $F(\tilde{\alpha})$ can be rewritten as:

$$
d \tilde{Y}=\frac{d F(\tilde{\alpha})}{d \tilde{\alpha}} \otimes d \tilde{\alpha}
$$

\section{Theorem}

4.

Let $\tilde{Y}=F(\tilde{\alpha})=\left(\left[f_{1}(a), f_{2}(b)\right],\left[g_{1}(c), g_{2}(d)\right]\right)$ be an IVIFF, if $F(\tilde{\alpha})$ has the subtraction derivative at $\tilde{\alpha}$, and $\tilde{\alpha}^{\prime} \in \tilde{S}^{\oplus}(\tilde{\alpha}, F)$, then we have

$$
F\left(\tilde{\alpha}^{\prime}\right) \ominus F(\tilde{\alpha}) \approx \frac{d F(\tilde{\alpha})}{d \tilde{\alpha}} \otimes\left(\tilde{\alpha}^{\prime} \ominus \tilde{\alpha}\right)
$$

that is $\Delta \tilde{Y} \approx d \tilde{Y}$, which satisfies the following conditions:

$$
\begin{array}{ll}
\lim _{\Delta a \rightarrow 0} \frac{A(\Delta \tilde{Y})-A(d \tilde{Y})}{\Delta a}=0, & \lim _{\Delta b \rightarrow 0} \frac{B(\Delta \tilde{Y})-B(d \tilde{Y})}{\Delta b}=0 \\
\lim _{\Delta c \rightarrow 0} \frac{C(\Delta \tilde{Y})-C(d \tilde{Y})}{\Delta c}=0, & \lim _{\Delta d \rightarrow 0} \frac{D(\Delta \tilde{Y})-D(d \tilde{Y})}{\Delta d}=0
\end{array}
$$

Proof. For any $\tilde{\alpha} \in \tilde{S}^{\oplus}(\tilde{\alpha}, F)$, we have

$$
\begin{aligned}
\Delta \tilde{\alpha} & =\tilde{\alpha}^{\prime} \ominus \tilde{\alpha}=\left(\left[a^{\prime}, b^{\prime}\right],\left[c^{\prime}, d^{\prime}\right]\right) \oplus([a, b],[c, d]) \\
& =\left(\left[\frac{a^{\prime}-a}{1-a}, \frac{b^{\prime}-b}{1-b}\right],\left[\frac{c^{\prime}}{c}, \frac{d^{\prime}}{d}\right]\right)
\end{aligned}
$$

and

$$
\begin{aligned}
\frac{d F(\tilde{\alpha})}{d \tilde{\alpha}}= & \left(\left[\frac{1-a}{1-f_{1}(a)} f_{1}^{\prime}(a), \frac{1-b}{1-f_{2}(b)} f_{2}^{\prime}(b)\right],\right. \\
& {\left.\left[1-\frac{c}{g_{1}(c)} g_{1}^{\prime}(c), 1-\frac{d}{g_{2}(d)} g_{2}^{\prime}(d)\right]\right) }
\end{aligned}
$$

So we can get

$$
\begin{aligned}
& d \tilde{Y}=\frac{d F(\tilde{\alpha})}{d \tilde{\alpha}} \otimes \Delta \tilde{\alpha}=\left(\left[\frac{a^{\prime}-a}{1-f_{1}(a)} f_{1}^{\prime}(a), \frac{b^{\prime}-b}{1-f_{2}(b)} f_{2}^{\prime}(b)\right],\right. \\
& \left.\left[1-\frac{c-c^{\prime}}{g_{1}(c)} g_{1}^{\prime}(c), 1-\frac{d-d^{\prime}}{g_{2}(d)} g_{2}^{\prime}(d)\right]\right) \\
& F(\tilde{\alpha}) \oplus\left(\frac{d F(\tilde{\alpha})}{d \tilde{\alpha}} \otimes \Delta \tilde{\alpha}\right)=\left(\left[f_{1}(a), f_{2}(b)\right],\left[g_{1}(c), g_{2}(d)\right]\right) \\
& \oplus\left(\left[\frac{a^{\prime}-a}{1-f_{1}(a)} f_{1}^{\prime}(a), \frac{b^{\prime}-b}{1-f_{2}(b)} f_{2}^{\prime}(b)\right],\left[1-\frac{c-c^{\prime}}{g_{1}(c)} g_{1}^{\prime}(c), 1-\frac{d-d^{\prime}}{g_{2}(d)} g_{2}^{\prime}(d)\right]\right) \\
& =\left(\left[f_{1}(a)+\left(a^{\prime}-a\right) f_{1}^{\prime}(a), f_{2}(b)+\left(b^{\prime}-b\right) f_{2}^{\prime}(b)\right]\right. \text {, } \\
& \left.\left[g_{1}(c)+\left(c^{\prime}-c\right) g_{1}^{\prime}(c), g_{2}(d)+\left(d^{\prime}-d\right) g_{2}^{\prime}(d)\right]\right) \\
& =\left(\left[f_{1}(a)+f_{1}^{\prime}(a)-f_{1}(a)+o\left(a^{\prime}-a\right), f_{2}(b)+f_{2}^{\prime}(b)-f_{2}(b)+o\left(b^{\prime}-b\right)\right]\right. \text {, } \\
& \left.\left[g_{1}(c)+g_{1}^{\prime}(c)-g_{1}(c)+o\left(c^{\prime}-c\right), g_{2}(d)+g_{2}^{\prime}(d)-g_{2}(d)+o\left(d^{\prime}-d\right)\right]\right) \\
& =\left(\left[f_{1}^{\prime}(a)+o\left(a^{\prime}-a\right), f_{2}^{\prime}(b)+o\left(b^{\prime}-b\right)\right]\right. \text {, } \\
& \left.\left[g_{1}^{\prime}(c)+o\left(c^{\prime}-c\right), g_{2}^{\prime}(d)+o\left(d^{\prime}-d\right)\right]\right) \\
& \approx F\left(\tilde{\alpha}^{\prime}\right)
\end{aligned}
$$

Thus, we have $F\left(\tilde{\alpha}^{\prime}\right) \ominus F(\tilde{\alpha}) \approx \frac{d F(\tilde{\alpha})}{d \tilde{\alpha}} \otimes\left(\tilde{\alpha}^{\prime} \ominus \tilde{\alpha}\right)$, that is, $\Delta \tilde{Y} \approx d \tilde{Y}$, and

$$
\begin{aligned}
& \lim _{\Delta a \rightarrow 0} \frac{A(\Delta \tilde{Y})-A(d \tilde{Y})}{\Delta a}=0, \lim _{\Delta b \rightarrow 0} \frac{B(\Delta \tilde{Y})-B(d \tilde{Y})}{\Delta b}=0 \\
& \lim _{\Delta c \rightarrow 0} \frac{C(\Delta \tilde{Y})-C(d \tilde{Y})}{\Delta c}=0, \lim _{\Delta d \rightarrow 0} \frac{D(\Delta \tilde{Y})-D(d \tilde{Y})}{\Delta d}=0
\end{aligned}
$$

Similarly, we have

$F(\tilde{\alpha}) \ominus F\left(\tilde{\alpha}^{\prime}\right) \approx \frac{d F(\tilde{\alpha})}{d \tilde{\alpha}} \otimes\left(\tilde{\alpha} \ominus \tilde{\alpha}^{\prime}\right)$ when $\tilde{\alpha}^{\prime} \in \tilde{S}^{\ominus}(\tilde{\alpha}, F)$.

Below, we compute the approximate values of 
some IVIFFs to demonstrate the effectiveness of Theorem 4:

Example 1. Let $\tilde{Y}=F(\tilde{\alpha})=\lambda \cdot \tilde{\alpha},(0<\lambda \leq 1)$, so $f_{1}(a)=1-(1-a)^{\lambda}, f_{2}(b)=1-(1-b)^{\lambda} \quad$ and $g_{1}(c)=c^{\lambda}, g_{2}(d)=d^{\lambda}$. By the definition of derivative of IVIFF, we have

$$
\frac{d F(\tilde{\alpha})}{d \tilde{\alpha}}=([\lambda, \lambda],[1-\lambda, 1-\lambda])
$$

Furthermore, by Theorem 4, we can get

$$
F(\tilde{\alpha} \oplus \Delta \tilde{\alpha}) \ominus F(\tilde{\alpha}) \approx([\lambda, \lambda],[1-\lambda, 1-\lambda]) \otimes \Delta \tilde{\alpha}
$$

On the other hand, by the operational laws of IVIFVs $\lambda\left(\tilde{\alpha}_{1} \oplus \tilde{\alpha}_{2}\right)=\lambda \tilde{\alpha}_{1} \oplus \lambda \tilde{\alpha}_{2}$, we have

$$
F(\tilde{\alpha} \oplus \Delta \tilde{\alpha}) \ominus F(\tilde{\alpha})=\lambda(\tilde{\alpha} \oplus \Delta \tilde{\alpha}) \ominus \lambda \tilde{\alpha}=\lambda \Delta \tilde{\alpha}
$$

Suppose that $\Delta \tilde{\alpha}=([0.01,0.02],[0.96,0.97])$, and $\lambda=0.3$, then

$$
\begin{aligned}
d \tilde{Y} & =([\lambda, \lambda],[1-\lambda, 1-\lambda]) \otimes \Delta \tilde{\alpha} \\
& =([0.003,0.006],[0.988,0.991]) \\
\Delta \tilde{Y}=\lambda \Delta \tilde{\alpha} & =([0.0030,0.0060],[0.9878,0.9909])
\end{aligned}
$$

From the above results, we can find that the value of $d \tilde{Y}$ is very close to the one of $\Delta \tilde{Y}$. problem:

Next, we consider a common decision making Example 2. Assume that three experts give their evaluation values using the IVIFVs: $\tilde{\alpha}_{1}=([0.2,0.3],[0.3,0.4]), \tilde{\alpha}_{2}=([0.1,0.2],[0.2,0.5])$ an $\mathrm{d} \tilde{\alpha}_{3}=([0.1,0.2],[0.2,0.3])$ for an alternative, and their weight vector is $\omega=(0.2,0.4,0.4)^{T}$. Using the IIFWA operator, we can calculate their overall value:

$$
\begin{aligned}
& \operatorname{IIFWA}\left(\tilde{\alpha}_{1}, \tilde{\alpha}_{2}, \tilde{\alpha}_{3}\right)= \\
& \left(\left[1-\prod_{j=1}^{3}\left(1-a_{j}\right)^{\omega_{j}}, 1-\prod_{j=1}^{3}\left(1-b_{j}\right)^{\omega_{j}}\right],\left[\prod_{j=1}^{3} c_{j}^{\omega_{j}}, \prod_{j=1}^{3} d_{j}^{\omega_{j}}\right]\right) \\
& =\left(\left[1-(1-0.2)^{0.2} \times(1-0.1)^{0.4} \times(1-0.1)^{0.4},\right.\right. \\
& \quad\left[1-(1-0.3)^{0.2} \times(1-0.2)^{0.4} \times(1-0.2)^{0.4}\right], \\
& \left.\quad\left[0.3^{0.2} \times 0.2^{0.4} \times 0.2^{0.4}, 0.4^{0.2} \times 0.5^{0.4} \times 0.3^{0.4}\right]\right)
\end{aligned}
$$

$=([0.121,0.221],[0.217,0.390])$

But if some of the experts, for example, the first expert would like to adjust the value of $\tilde{\alpha}_{1}$ slightly and gives the new assessment $\tilde{\alpha}_{1}^{\prime}$, then we can deal with the information aggregation as follows:

If $\tilde{\alpha}_{1}^{\prime} \in \tilde{S}^{\oplus}\left(\tilde{\alpha}_{1}, F\right)$, then there must exist an IVIFV $\tilde{\beta}_{1} \quad$ such that $\tilde{\alpha}_{1}^{\prime}=\tilde{\alpha}_{1} \oplus \tilde{\beta}_{1}$. Assume that $\tilde{\alpha}_{1}^{\prime}=([0.3,0.4],[0.2,0.3]) \in \tilde{S}^{\oplus}\left(\tilde{\alpha}_{1}, F\right) \quad, \quad$ then $\tilde{\beta}_{1}=\tilde{\alpha}_{1}^{\prime} \ominus \tilde{\alpha}_{1}=([0.125,0.143],[0.667,0.75])$

and

$$
\begin{aligned}
& \operatorname{IIFWA}\left(\tilde{\alpha}_{1}^{\prime}, \tilde{\alpha}_{2}, \tilde{\alpha}_{3}\right) \approx I I F W A_{\omega}\left(\tilde{\alpha}_{1}, \tilde{\alpha}_{2}, \tilde{\alpha}_{3}\right) \\
& \oplus\left(\left[\omega_{1}, \omega_{1}\right],\left[1-\omega_{1}, 1-\omega_{1}\right]\right) \otimes\left(\tilde{\alpha}_{1}^{\prime} \ominus \tilde{\alpha}_{1}\right) \\
& =([0.121,0.221],[0.217,0.390]) \oplus \\
& ([0.2,0.2],[0.8,0.8]) \otimes([0.125,0.143],[0.667,0.75]) \\
& =([0.144,0.245],[0.2,0.368])
\end{aligned}
$$

Based on the division derivative, we shall define the other kind of differential--the division differential:

Definition 31. Suppose that $\tilde{Y}=F(\tilde{\alpha})=\left(\left[f_{1}(a), f_{2}(b)\right],\left[g_{1}(c), g_{2}(d)\right]\right) \quad$ is $\quad$ an IVIFF and $\nabla \tilde{\alpha}=\tilde{\alpha}^{\prime} \oslash \tilde{\alpha}=\frac{\tilde{\alpha}^{\prime}}{\tilde{\alpha}}$, then the division differential of $F(\tilde{\alpha})$ is defined as $\frac{l F(\tilde{\alpha})}{l \tilde{\alpha}} \oplus \nabla \tilde{\alpha}$, denoted by $l \tilde{Y}$, that is,

$$
l \tilde{Y}=\frac{l F(\tilde{\alpha})}{l \tilde{\alpha}} \oplus \nabla \tilde{\alpha}
$$

Similarly, for the identity function $F(\tilde{\alpha})=\tilde{\alpha}=([a, b],[c, d])$, when computing its division differential, we have $l \tilde{\alpha}=\nabla \tilde{\alpha}$, so the division differential can be rewritten as:

$$
l \tilde{Y}=\frac{l F(\tilde{\alpha})}{l \tilde{\alpha}} \oplus l \tilde{\alpha}
$$

Theorem

5. Let $\tilde{Y}=F(\tilde{\alpha})=\left(\left[f_{1}(a), f_{2}(b)\right],\left[g_{1}(c), g_{2}(d)\right]\right)$ be an IVIFF, if $F(\tilde{\alpha})$ owns the division derivative at $\tilde{\alpha}$, and $\tilde{\alpha}^{\prime} \in \tilde{S}^{\otimes}(\tilde{\alpha}, F)$, then we get 


$$
F\left(\tilde{\alpha}^{\prime}\right) \oslash F(\tilde{\alpha}) \approx \frac{l F(\tilde{\alpha})}{l \tilde{\alpha}} \oplus\left(\tilde{\alpha}^{\prime} \oslash \tilde{\alpha}\right)
$$

Noting that $\nabla \tilde{Y}=F\left(\tilde{\alpha}^{\prime}\right) \oslash F(\tilde{\alpha})=\frac{F\left(\tilde{\alpha}^{\prime}\right)}{F(\tilde{\alpha})} \quad$ and $\nabla \tilde{\alpha}=\tilde{\alpha}^{\prime} \oslash \tilde{\alpha}$, we have $\nabla \tilde{Y} \approx l \tilde{Y}$, satisfying the following conditions:

$$
\begin{aligned}
& \lim _{\Delta a \rightarrow 0} \frac{A(\Delta \tilde{Y})-A(l \tilde{Y})}{\Delta a}=0, \lim _{\Delta b \rightarrow 0} \frac{B(\Delta \tilde{Y})-B(l \tilde{Y})}{\Delta b}=0 \\
& \lim _{\Delta c \rightarrow 0} \frac{C(\Delta \tilde{Y})-C(l \tilde{Y})}{\Delta c}=0, \lim _{\Delta d \rightarrow 0} \frac{D(\Delta \tilde{Y})-D(l \tilde{Y})}{\Delta d}=0
\end{aligned}
$$

When $\tilde{\alpha}^{\prime} \in \tilde{S}^{\ominus}(\tilde{\alpha}, F)$, we have

$$
F(\tilde{\alpha}) \oslash F\left(\tilde{\alpha}^{\prime}\right) \approx \frac{l F(\tilde{\alpha})}{l \tilde{\alpha}} \oplus\left(\tilde{\alpha} \oslash \tilde{\alpha}^{\prime}\right)
$$

Example 3. If the IVIFF $F(\tilde{\alpha})=\tilde{\alpha}^{\lambda},(0<\lambda \leq 1)$, that is, $f_{1}(a)=a^{\lambda}, f_{2}(b)=b^{\lambda} \quad, \quad g_{1}(a)=1-(1-a)^{\lambda}$,
$g_{2}(b)=1-(1-b)^{\lambda}$, and

$$
\frac{l F(\tilde{X})}{l \tilde{X}}=([1-\lambda, 1-\lambda],[\lambda, \lambda])
$$

By Theorem 5, we have

$$
\frac{F(\tilde{\alpha} \otimes \nabla \tilde{\alpha})}{F(\tilde{\alpha})} \approx([1-\lambda, 1-\lambda],[\lambda, \lambda]) \oplus \nabla \tilde{\alpha}
$$

Because $\left(\tilde{\alpha}_{1} \otimes \tilde{\alpha}_{2}\right)^{\lambda}=\tilde{\alpha}_{1}^{\lambda} \otimes \tilde{\alpha}_{2}{ }^{\lambda}$, then we have

$$
\frac{F(\tilde{\alpha} \otimes \nabla \tilde{\alpha})}{F(\tilde{\alpha})}=\frac{(\tilde{\alpha} \otimes \nabla \tilde{\alpha})^{\lambda}}{\tilde{\alpha}^{\lambda}}=(\nabla \tilde{\alpha})^{\lambda}
$$

If we suppose $\nabla \tilde{\alpha}=([0.90,0.92],[0.03,0.05])$ and $\lambda=0.4$, then we can get

$([1-\lambda, 1-\lambda],[\lambda, \lambda]) \oplus \nabla \tilde{\alpha}=([0.96,0.968],[0.012,0.02])$ and

$$
(\nabla \tilde{\alpha})^{\lambda}=([0.959,0.967],[0.012,0.020])
$$

which shows that the approximate degree of replacing $(\nabla \tilde{\alpha})^{\lambda}$ with $([1-\lambda, 1-\lambda],[\lambda, \lambda]) \oplus \nabla \tilde{\alpha}$ is very high.

From the proof process of Theorem 4, we can further derive the following conclusion:

\section{Theorem}

6.

$\tilde{Y}=F(\tilde{\alpha})=\left(\left[f_{1}(a), f_{2}(b)\right],\left[g_{1}(c), g_{2}(d)\right]\right)$ be an
IVIFF which satisfies the conditions: $f_{1}^{\prime \prime}(a)=f_{2}^{\prime \prime}(b)=g_{1}^{\prime \prime}(c)=g_{2}^{\prime \prime}(d)=0$ and $\tilde{\alpha}^{\prime} \in \tilde{S}^{\oplus}(\tilde{\alpha}, F)$, then

$$
F\left(\tilde{\alpha}^{\prime}\right) \ominus F(\tilde{\alpha})=\frac{d F(\tilde{\alpha})}{d \tilde{\alpha}} \otimes\left(\tilde{\alpha}^{\prime} \ominus \tilde{\alpha}\right)
$$

The same holds true for $\tilde{\alpha}^{\prime} \in \tilde{S}^{\ominus}(\tilde{\alpha}, F), \tilde{\alpha}^{\prime} \in \tilde{S}^{\otimes}(\tilde{\alpha}, F)$ or $\tilde{\alpha}^{\prime} \in \tilde{S}^{\ominus}(\tilde{\alpha}, F)$.

Proposition 1 in Section 2 has shown several operation laws for IVIFVs, such as the commutative law and the distributive law between the scalar multiplication and addition operations. But the existence of the distributive laws between the multiplication and addition operations is still unknown. In the following, we shall discuss this question:

Example 4. Assume $\tilde{\alpha}_{0}=\left(\left[a_{0}, b_{0}\right],\left[c_{0}, d_{0}\right]\right)$, $\tilde{\alpha}=([a, b],[c, d])$ and $\tilde{\alpha}^{\prime}=\left(\left[a^{\prime}, b^{\prime}\right],\left[c^{\prime}, d^{\prime}\right]\right)$ are three arbitrary IVIFVs, and let the IVIFF $F(\tilde{\alpha})=\tilde{\alpha}_{0} \otimes \tilde{\alpha}$, in which $f_{1}(a)=a_{0} a, f_{2}(b)=b_{0} b \quad$ and $g_{1}(c)=c_{0}+c-c_{0} c, g_{2}(d)=d_{0}+d-d_{0} d$. Obviously, $f_{1}^{\prime \prime}(a)=f_{2}^{\prime \prime}(b)=g_{1}^{\prime \prime}(c)=g_{2}^{\prime \prime}(d)=0$. So by Theorem 6 , we have

$$
F\left(\tilde{\alpha} \oplus \tilde{\alpha}^{\prime}\right) \ominus F(\tilde{\alpha})=\frac{d F(\tilde{\alpha})}{d \tilde{\alpha}} \otimes \tilde{\alpha}^{\prime}
$$

That is, $F\left(\tilde{\alpha} \oplus \tilde{\alpha}^{\prime}\right)=F(\tilde{\alpha}) \oplus \frac{d F(\tilde{\alpha})}{d \tilde{\alpha}} \otimes \tilde{\alpha}^{\prime}$.

By the example in Section 5, we can get

$$
\begin{aligned}
& \frac{d F(\tilde{\alpha})}{d \tilde{\alpha}}=\tilde{\alpha}_{0} \otimes\left(\left[\frac{1-a}{1-a_{0} a}, \frac{1-b}{1-b_{0} b}\right],\right. \\
& \left.\left[1-\frac{c}{c_{0}+c-c_{0} c}, 1-\frac{d}{d_{0}+d-d_{0} d}\right]\right)
\end{aligned}
$$

Then the following equation holds:

$$
\begin{aligned}
& \tilde{\alpha}_{0} \otimes\left(\tilde{\alpha} \oplus \tilde{\alpha}^{\prime}\right)=\tilde{\alpha}_{0} \otimes \tilde{\alpha} \oplus \tilde{\alpha}_{0} \otimes \tilde{\alpha}^{\prime} \otimes \\
& \left(\left[\frac{1-a}{1-a_{0} a}, \frac{1-b}{1-b_{0} b}\right],\left[1-\frac{c}{c_{0}+c-c_{0} c}, 1-\frac{d}{d_{0}+d-d_{0} d}\right]\right)
\end{aligned}
$$

The above equation shows that $\tilde{\alpha}_{0} \otimes\left(\tilde{\alpha} \oplus \tilde{\alpha}^{\prime}\right) \neq \tilde{\alpha}_{0} \otimes \tilde{\alpha} \oplus \tilde{\alpha}_{0} \otimes \tilde{\alpha}^{\prime} \quad, \quad$ i.e., $\quad$ the distributive laws between the multiplication and addition operations is not correct. 


\section{Conclusions}

In this paper, we have firstly presented the concepts of the change values of IVIFVs, based on which, we have classified the sequences of IVIFVs and given the limit definitions of these sequences respectively. Moreover, we have introduced the concept of IVIFF. After all these preparations, we have studied the continuities and derivatives (the subtraction and division derivatives) of IVIFFs. To make the concepts of derivatives of IVIFFs easier to be understood, we have illustrated them by some special IVIFFs. Based on the derivatives proposed previously, at the end of the paper, we have investigated two differential operations (the subtraction and division differentials) of IVIFFs and applied them in estimating the values of IVIFFs.

\section{Acknowledgments}

The work was supported by the National Natural Science Foundation of China (No.61273209) and the Central University Basic Scientific Research Business Expenses Project (No. skgt201501). The author also would like to thank the editor and the reviewers whose helpful comments have led to the improvements of the paper.

\section{References}

1. Zadeh LA. Fuzzy sets. Information and Control 1965;8(3): 338-353. DOI:10.1016/S0019-9958(65)90241-X

2. Atanassov K. Intuitionistic fuzzy sets. Fuzzy Sets and Systems 1986;20(1):87-96. DOI:10.1016/S01650114(86)80034-3

3. Szmidt E., Kacprzyk J. Using intuitionistic fuzzy sets in group decision making. Control and Cybernetics 2002;31(4):1037-1053.

4. Xu ZS. Intuitionistic fuzzy aggregation operators. IEEE Transactions on Fuzzy Systems 2007;15(6):1179-1187. DOI: 10.1109/TFUZZ.2006.890678

5. $\mathrm{Xu}$ ZS. Intuitionistic preference relations and their application in group decision making. Information Sciences 2007;177(11):2363-2379. DOI:10.1016/j.ins.2006.12.019

6. Zhao H., Xu ZS., Liu SS., Wang Z. Intuitionistic fuzzy MST clustering algorithms. Computers \& Industrial Engineering 2012;62(4):1130-1140. DOI: 10.1016/j.cie.2012.01.007

7. Wang Z., Xu ZS., Liu SS., Yao ZQ. Direct clustering analysis based on intuitionistic fuzzy implication. Applied Soft Computing 2014;23:1-8. DOI: 10.1016/j.asoc.2014.03.037
8. Le HS., Nguyen TT. Intuitionistic fuzzy recommender systems: An effective tool for medical diagnosis. Knowledge-Based Systems 2015;74:133-150. DOI: 10.1016/j.knosys.2014.11.012

9. Balasubramaniam P., Ananthi V.P. Image fusion using intuitionistic fuzzy sets. Information Fusion 2014;20:2130. DOI: 10.1016/j.knosys.2014.11.012

10. Da Costa CG., Bedregal B., Neto ADD. Atanassov's intuitionistic fuzzy probability and Markov chains. Knowledge-Based Systems 2013;43:52-62. DOI: 10.1016/j.knosys.2013.01.015

11. Zheng MC., Shi ZK., Liu Y. Triple I method of approximate reasoning on Atanassov's intuitionistic fuzzy sets. International Journal of Approximate Reasoning 2014;55(6):1369-1382. DOI: 10.1016/j.ijar.2014.01.001

12. Dawaz B., Dudek WA., Jun YB. Intuitionistic fuzzy H-vsubmodules. Information Sciences 2006;176(3):285-300. DOI: $10.1016 /$ j.ins.2004.10.009

13. Chen WJ., Zhang SH. Intuitionistic fuzzy Lie sub-super algebras and intuitionistic fuzzy ideals. Computers and Mathematics with Applications 2009;58(8):1645-1661. DOI: $10.1109 /$ CSO.2009.399

14. Lei Q., Xu ZS. Derivative and differential operations of intuitionistic fuzzy numbers. International Journal of Intelligent Systems 2015;30(4):468-498. DOI: 10.1002/int.21696

15. Lei Q., Xu ZS. Fundamental properties of intuitionistic fuzzy calculus. Knowledge-Based Systems 2015;76:1-16. DOI:10.1016/j.knosys.2014.11.019

16. Lei Q., Xu ZS., Bustince H., Fernandez J. Intuitionistic fuzzy integrals based on Archimedean t-conorms and tnorms, Information Sciences 2016;327:57-70. DOI: 10.1016/j.ins.2015.08.005

17. $\mathrm{Xu}$ ZS., Yager RR. Some geometric aggregation operators based on intuitionistic fuzzy sets. International Journal of General Systems 2006;35(4):417-433. DOI:10.1080/03081070600574353

18. Yu XH., Xu ZS., Liu SS., Chen Q. On ranking of intuitionistic fuzzy values based on dominance relations. International Journal of Uncertainty, Fuzziness and Knowledge-Based Systems 2014;22(2):315-335. DOI: $10.1142 / \mathrm{S} 0218488514500160$

19. Guo KH. Amount of information and attitudinal-based method for ranking Atanassov's intuitionistic fuzzy values. IEEE Tansactions on Fuzzy Systems 2014;22(1): 177-188. DOI:10.1109/TFUZZ.2013.2249586

20. Zhang XM., Xu ZS. A new method for ranking intuitionistic fuzzy values and its application in multiattribute decision making. Fuzzy Optimization and Decision Making 2012;11(2):135-146. DOI: 10.1007/s10700-012-9118-9

21. Atanassov K., Gargov G. Interval-valued intuitionistic fuzzy sets. Fuzzy Sets and Systems 1989;31(3):343-349. DOI: 10.1016/0165-0114(89)90205-4

22. Jin FF., Pei LD., Chen HY., Zhou LG. Interval-valued intuitionistic fuzzy continuous weighted entropy and its 
application to multi-criteria fuzzy group decision making. Knowledge-Based Systems 2014;59:132-141. DOI:10.1016/j.knosys.2014.01.014

23. Chen TY. The extended linear assignment method for multiple criteria decision analysis based on intervalvalued intuitionistic fuzzy sets. Applied Mathematical Modeling 2014;38(7-8):2101-2117. DOI: 10.1016/j.apm.2013.10.017

24. Meng FY., Tan CQ., Zhang Q. The induced generalized interval-valued intuitionistic fuzzy hybrid Shapley averaging operator and its application in decision making. Knowledge-Based Systems 2013;42(2):9-19. DOI: 10.1016/j.knosys.2012.12.006

25. Zhao H., Xu ZS. Group decision making with densitybased aggregation operators under interval-valued intuitionistic fuzzy environments. Journal of Intelligent and Fuzzy Systems 2014;27(2):1021-1033. DOI: $10.3233 /$ IFS-131063

26. Li DF., Linear programming method for MADM with interval-valued intuitionistic fuzzy sets. Expert Systems with Applications 2010;37(8):5939-5945. DOI:10.1016/j.eswa.2010.02.011

27. Wu J., Huang HB., Cao QW. Research on AHP with interval-valued intuitionistic fuzzy sets and its application in multi-criteria decision making problems. Applied Mathematical Modelling 2013;37(24):9898-9906. DOI:10.1016/j.apm.2013.05.035

28. $\mathrm{Xu} \mathrm{ZS}$. Methods for aggregating interval-valued intuitionistic fuzzy information and their application to decision making. Control and Decision 2007;22(2):215219. DOI:10.3321/j.issn:1001-0920.2007.02.020

29. Wu J., Chiclana F. A risk attitudinal ranking method for interval-valued intuitionistic fuzzy numbers based on novel attitudinal expected score and accuracy functions. Applied Soft Computing 2014;22(5):272-286. DOI:10.1016/j.asoc.2014.05.005

30. Zhang ZH., Yang JY., Ye YP., Zhang QS. A generalized interval-valued intuitionistic fuzzy sets. Procedia Engineering 2011;15:2037-2041. DOI:10.1016/j.proeng.2011.08.380

31. Deschrijver G., Kerre EE. On the relationship between some extensions of fuzzy set theory. Fuzzy Sets and Systems 2003;133(2):227-235. DOI: 10.1016/S01650114(02)00127-6 